\title{
Dynamical arrest with zero complexity: the unusual behavior of the spherical Blume Emery Griffiths disordered model
}

\author{
Corrado Rainone ${ }^{1,2}$, Ulisse Ferrari ${ }^{3}$, Matteo Paoluzzi ${ }^{1}$ and Luca Leuzzi ${ }^{4,1}$ \\ 1 Dipartimento di Fisica, Sapienza Università di Roma, Piazzale A. Moro 2, I-00185 Roma, Italy \\ 2 LPT, Ecole Normale Supérieure, CNRS UMR 8549, 24 Rue Lhomond, 75005 Paris, France \\ 3 Institut de la Vision, Sorbonne Universités, UPMC, \\ INSERM U968, CNRS UMR 7210, Paris, F-75012, France \\ 4 NANOTEC-CNR, Soft and Living Matter Lab. Rome, c/o Dept. Physics, \\ Sapienza Università di Roma, Piazzale A. Moro 2, I-00185 Roma, Italy
}

(Dated: October 25, 2018)

\begin{abstract}
The short- and long-time dynamics of model systems undergoing a glass transition with apparent inversion of Kauzmann and dynamical arrest glass transition lines is investigated. These models belong to the class of the spherical mean-field approximation of a spin-1 model with $p$-body quenched disordered interaction, with $p>2$, termed spherical Blume-Emery-Griffiths models. Depending on temperature and chemical potential the system is found in a paramagnetic or in a glassy phase and the transition between these phases can be of a different nature. In specific regions of the phase diagram coexistence of low density and high density paramagnets can occur, as well as the coexistence of spin-glass and paramagnetic phases. The exact static solution for the glassy phase is known to be obtained by the one-step replica symmetry breaking ansatz. Different scenarios arise for both the dynamic and the thermodynamic transitions. These include: (i) the usual random first- order transition (Kauzmann-like) for mean-field glasses preceded by a dynamic transition, (ii) a thermodynamic first-order transition with phase coexistence and latent heat and (iii) a regime of apparent inversion of static transition line and dynamic transition lines, the latter defined as a nonzero complexity line. The latter inversion, though, turns out to be preceded by a novel dynamical arrest line at higher temperature. Crossover between different regimes is analyzed by solving mode coupling theory equations throughout the space of external thermodynamic parameters and the relationship with the underlying statics is discussed.
\end{abstract}

\section{INTRODUCTION}

In the present work we investigate the dynamic properties of a glassy system in which, under certain external conditions, both glass and fluid can coexists, yielding different scenarios for dynamical arrest and for the fluidglass transition. These properties can be studied in statistical mechanical models with bosonic spin-1 variables, where the holes $s=0$ play the role of inactive states, that is, the so-called Blume-Capel [1, 2] or Blume-Emery Griffiths (BEG) [3] models. In these models the fluid phase corresponds to a paramagnet and the solid phase is either a ferromagnet (no or weak disorder) [1-5] or a spin glass (strong disorder) [6-12]. In the present work we consider an extension to $p$-spin interacting systems with spin-1, to $p>2$ and continuous (spherical) variables [13] to better represent continuous density fluctuations, alike to liquid-like compounds.

In the presence of quenched disorder the random BEG model with pairwise $(p=2)$, as well as its spherical counterpart, is known to display both a continuous paramagnet/spin-glass phase transition and a first-order one (first order in the thermodynamic sense, i.e. with latent heat and a region of phase coexistence). Furthermore, melting upon cooling [14-20] can occur, with a spin glass at high $T$ and a paramagnet at low $T$. These properties have been observed in the mean-field approximation, where the self-consistent solution for the spin-glass phase is computed in the full replica symmetry breaking
(RSB) Parisi ansatz [21] and on the cubic 3D lattice with nearest-neighbor couplings [22, 23]. The frustrated BEG model has been studied by means of numerical renormalization group techniques, as well, with results depending on the underlying lattice and the renormalization technique adopted [24-26].

Mean-field spin-glass models with Ising [27], soft [28, 29 ] or spherical $[30,31]$ spins with more than two-spin interactions, called $p$-spin models, are known to yield the so-called random first-order transition, i.e., a phase transition across which no internal energy discontinuity occurs but the order parameter (the Edwards-Anderson overlap $q_{E A}$ ) jumps from zero to a finite value. Their glassy phase is described by an ansatz with one RSB [32]. In a cooling procedure, the thermodynamic transition is preceded by a dynamic transition due to the onset of a very large number of metastable states separated by high barriers [33]. "Very large" means that the number of states $\mathcal{N}$ grows exponentially with the size $N$ of the system: $\mathcal{N} \sim \exp (\Sigma N)$ where the coefficient $\Sigma$ is the configurational entropy, also called complexity in the framework of spin-glass systems (see, e.g, Refs. [34, 35] and references therein). "High barriers" means that the free energy difference between a local minimum in the free energy functional of the configurational space (also called free energy landscape) and a nearby maximum (or saddle) grows with $N$. The phenomenology of the $p$-spin spin-glass systems is, in many respects, very similar to the one of structural glasses. These models are, therefore, sometimes called mean-field glasses. The occurrence 
of non-zero $\Sigma$ is a fundamental property both in meanfield systems $[28,29,36]$ and outside the range of validity of mean-field theory, e.g. in computer glass models $[37,38]$, or, indirectly, by measuring the excess entropy of glasses in experiments, see, e.g., Ref. [33] and references therein. The barriers' height turns out to diverge in the thermodynamic limit in the mean-field approximation, this being an artifact of mean-field glasses. The thus induced dynamic transition corresponds to the transition predicted by another mean-field theory for the dynamics of supercooled liquids: the mode coupling theory [39]. The thermodynamic transition occurring at a lower temperature is, instead, the mean-field equivalent of the so-called Kauzmann transition in glasses, also known as the ideal glass transition [33]. This was initially predicted by Gibbs and Di Marzio [40] and its occurrence in real strutural glasses is still object of an ongoing debate [4144].

We are going to investigate the complex dynamic properties consequent to the combination of a kind of interaction inducing structural glass behavior and the presence of hole states (aka, spin state $s=0$ ) inducing phase coexistence. The latter element is, possibly, responsible for melting upon cooling [21, 22]. The first of such models was brought about by Sellitto in the pairwise random orthogonal model with spin-1 variables [45]. In the present dynamic work we rather consider the multi-body interaction model of Ref. [13], where both high temperature coexistence of high- and low-density paramagnetic phases, and low temperature coexistence of (low-density) paramagnetic and spin-glass phases are displayed.

\section{MODEL}

The model we consider is a spherical Blume-Capel $[1,2]$ model with $p$-body disordered interactions. Our starting point is the model Hamiltonian

$$
H=-\sum_{i_{1}<i_{2}<\cdots<i_{p}} J_{i_{1} i_{2} \ldots i_{p}} s_{i_{1}} s_{i_{2}} \ldots s_{i_{p}}+D \sum_{i} s_{i}^{2}
$$

where the variables $s_{i}$ are bosonic spins (i.e., $s_{i}=$ $1,0,-1)$, and the couplings are independent quenched random variables distributed with a Gaussian probability density,

$$
P\left(J_{i_{1} i_{2} \ldots i_{p}}\right)=\sqrt{\frac{N^{p-1}}{\pi J^{2} p !}} \exp \left[-\frac{J_{i_{1} i_{2} \ldots i_{p}}^{2} N^{p-1}}{p !}\right]
$$

the external parameter $D$ is called "crystal field" in literature, and it essentially plays the role of a chemical potential. Because of the bosonic spins, we cannot define the continous spin approximation of the model with spherical constraint in the usual way [46]. We must first rewrite the Hamiltonian as an Ising-spin problem on a lattice-gas

$$
\begin{aligned}
H= & -\sum_{i_{1}<i_{2}<\cdots<i_{p}} J_{i_{1} i_{2} \ldots i_{p}} n_{i_{1}} \sigma_{i_{1}} n_{i_{2}} \sigma_{i_{2}} \ldots n_{i_{p}} \sigma_{i_{p}} \\
& +(D-T \log 2) \sum_{i} n_{i}
\end{aligned}
$$

where the $-T \log 2$ term is necessary to keep the ratio of filled-in to empty sites identical to the one of the original Hamiltonian, see [47, 48] for details. We then introduce the variable[49]

$$
\tau_{i}=\sigma_{i}\left(2 n_{i}-1\right)= \pm 1
$$

This way, the model Hamiltonian assumes the form

$$
\begin{aligned}
H= & -\frac{1}{2^{p}} \sum_{i_{1}<i_{2}<\cdots<i_{p}} J_{i_{1} i_{2} \ldots i_{p}}\left(\sigma_{i_{1}}+\tau_{i_{1}}\right) \ldots\left(\sigma_{i_{p}}+\tau_{i_{p}}\right) \\
& +(D-T \log 2) \sum_{i=1}^{N} \frac{\sigma_{i} \tau_{i}+1}{2}
\end{aligned}
$$

where all the degrees of freedom, $\sigma$ 's and $\tau$ 's are now Ising spins. A continuous spin model can then be constructed by imposing two independent spherical constraints

$$
\sum_{i=1}^{N} \sigma_{i}^{2}=N, \quad \sum_{i=1}^{N} \tau_{i}^{2}=N
$$

Programma/Pspinbosonic/Test/ The thermodynamic properties of the model we just defined are thoroughly studied, applying 1RSB theory, in reference [13]. For the sake of brevity, henceforth we shall refer to Ref. [13] as the static study (that is, replica theory-based), in contrast with the dynamic study that constitutes the principal aim and subject of this paper. Before deriving and solving the dynamical equations of the model, it is suitable to briefly summarize the static results of [13], with particular emphasis on the aspects that will be most relevant for the dynamical study that we are going to report.

\section{A. The static phase diagram}

The 1RSB free energy for the model is

$$
\begin{aligned}
\beta F= & (\beta D-\log 2) d-\frac{\beta^{2}}{4}\left(d^{p}+(m-1) q_{1}^{p}\right)-\log 2 \\
& -\frac{1}{2}\left(\log (1-d)+\frac{m-1}{m} \log \eta_{0}+\frac{1}{m} \log \eta_{1}\right),
\end{aligned}
$$

with the definitions

$$
\begin{aligned}
& \eta_{0} \equiv d-q_{1}, \\
& \eta_{1} \equiv d+(m-1) q_{1}-m q_{0},
\end{aligned}
$$

where the parameter $d$ is the ratio of filled-in to empty sites (that is, the density of the system), and the $q_{0}$ and 
$q_{1}$ are respectively the mutual overlap and self-overlap, as usual in a 1RSB Ansatz. The extremization of the (6) whith respect to $q_{1}$ and $d$ yields the saddle-point equations

$$
\begin{aligned}
\frac{p \beta^{2}}{2} q_{1}^{p-1} & =\frac{q_{1}}{\eta_{0} \eta_{1}} \\
\frac{p \beta^{2}}{2} d^{p-1}-\frac{p \beta^{2}}{2} q_{1}^{p-1} & =\frac{\eta_{0}-\theta}{\theta \eta_{0}}+2(\beta D-\log 2) .
\end{aligned}
$$

The static lines of the phase diagram, in absence of an external magnetic field, can be determined by setting $q_{0}=0$ and studying the system given by the equations (8a), (8b), and the saddle-point condition for the $1 \mathrm{RSB}$ parameter $m$

$$
z(y)=\frac{2}{p},
$$

where $z(y)$ is the Crisanti-Sommers function[30]

$$
z(y) \equiv-2 y \frac{1-y+\log y}{(1-y)^{2}}, \quad y \equiv \frac{\eta_{0}}{\eta_{1}} .
$$

The dynamical arrest lines can be, as well, identified using replica theory, by solving the saddle-point equations for $m=1$, and by imposing the following marginality condition for the solution in the $d, q_{1}$ parameter space:

$$
\left|\begin{array}{cc}
\frac{\partial^{2} F}{\partial q_{1}^{2}} & \frac{\partial^{2} F}{\partial q_{1} \partial d} \\
\frac{\partial^{2} F}{\partial d \partial q_{1}} & \frac{\partial^{2} F}{\partial d^{2}}
\end{array}\right|=0 .
$$

This corresponds to looking for a spinodal point in the free energy as a function of $d$ and $q_{1}$.

The resulting phase diagram for the model is reported in figure 1.

From it, it can be seen that the system exhibits a rich phenomenology, with both random first-order transitions (RFOT) and thermodynamic first-order phase transitions (TFOPT). Here we will just comment on them briefly to adequately introduce our dynamical study, along with statics novelties with respect to previous analysis. The interested reader can find all the details in [13].

a. Random first-order transition For low enough $D$, the system exhibits the random first-order phenomenology typical of the $p$-spin model. Along the dynamic transition line $T_{d}(D)$, the system undergoes a dynamical arrest, meaning that the relaxation towards the paramagnetic, stable state is blocked by the presence of an exponentially large number of metastable SG states which trap the dynamics. Being the SG states metastable, this transition is not captured by the static saddle-point equations, and has to be studied by solving the dynamics of the model, or by using the marginality condition for the dynamics [30, 47, 50, 51].

At a temperature $T_{s}(D)$ lower than $T_{d}(D)$, a static transition takes place, whereupon the number of $\mathrm{SG}$ states becomes subexponential (their complexity, also
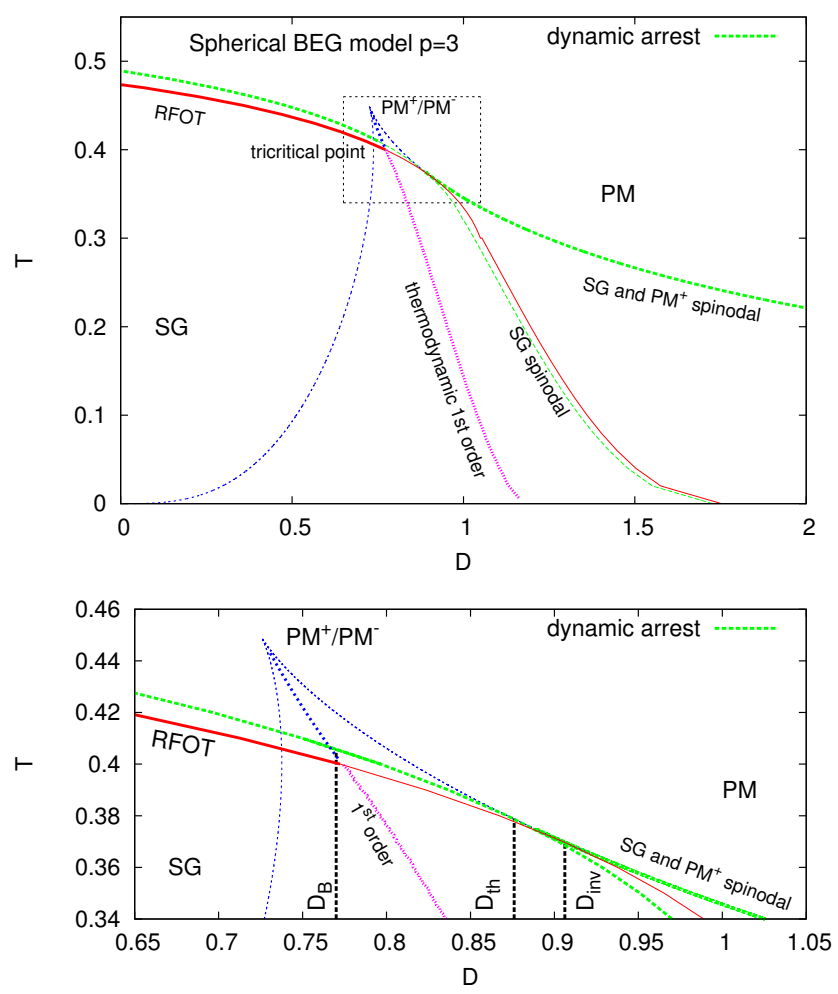

FIG. 1. The complete phase diagram for the model (4) for $p=3$. In the bottom inset the detail contained in the box of the main panel is displayed, together with he values of $D_{B}$, $D_{t h}$ e $D_{\text {inv }}$ (see text) identified by vertical dotted lines.

called configurational entropy, vanishes) and they become stable with respect to the paramagnet, yielding the equilibrium SG solution $[30,52]$. This feature of the model, occurring for $D<D_{B}=0.77$, is equivalent to the $p$-spin model phenomenology.

b. Thermodynamic first-order phase transitions In the region of the phase diagram between the spinodal lines, but above the RFOT line, two paramagnetic phases, termed $\mathrm{PM}^{-}$and $\mathrm{PM}^{+}$, coexist, both with $q_{1}=0$ but with two different density values $d$ : $d^{-}$and $d^{+}>d^{-}$. The two paramagnets are labelled + and - according to their density value $d$ being, respectively, large and small. These values can be determined by solving the saddlepoint Eq. (8b) in the $q_{1}=0$ limit, yielding the expression

$$
\frac{p}{2} d^{p}(1-d)=T^{2}(2 d-1)+2\left(T D-T^{2} \log 2\right)(1-d) d
$$

For $p=3$, this is a polynomial equation with three solutions for $d \in[0: 1]$. The solution with the intermediate value of $d$ turns out to be always unstable (see Ref. [13]), leaving only a high-density and a low-density solutions. Since the density $d$ has a continuous behavior along the PM-/SG thermodynamic transition, Eq. (12) can be used, as well, to determine the value of the density for the SG phase at the transition point. This 
means that the spinodal lines $T(D)$ can be determined in a parametric form in $d$ as

$$
\begin{aligned}
D_{s p}(d) & =\sqrt{\frac{p}{p-1}} \frac{d^{\frac{p}{2}-1}}{2(d-1) \sqrt{4 d^{2}-4 d+2}} \times \\
& \left\{d^{3}(p-1) 2 \log (2)+d^{2}[p(2-4 \log (2))+4 \log (2)]\right. \\
& +d[p(\log (4)-3)+1-2 \log (2)]+p\} \\
T_{s p}(d) & =\frac{(d-1) \sqrt{p(p-1)} d^{p / 2}}{\sqrt{4 d^{2}-4 d+2}} .
\end{aligned}
$$

By studying those expressions, it can be readily checked that for $p \geq 3$, at high $D$ the spinodal curve is an asymptote of the $D$ axis; this means that, however large $D$, the system can always present an high density phase, if the temperature is low enough. This fact will be of capital importance in the following of this paper.

Besides the RFOT, thus, the system also exhibits both a $\mathrm{PM}-/ \mathrm{PM}+$ and, furthermore, a PM-/SG thermodynamic first order transition, that means standard first order transitions with phase coexistence and latent heat. Both transitions take place along the TFOPT line in figure 1 and coexisting phases exist between the spinodal lines. As the temperature is decreased to cross the RFOT dynamic line, we observe that only the high-density paramagnet undergoes dynamical arrest, while the low density $\mathrm{PM}^{-}$phase is unperturbed. Thus, at $T$ lower than the crossing point of TFOPT and RFOT lines, the transition occurs between a low density paramagnet PM-, with $d=d_{-}$and $q_{1}=0$, and an high-density SG with $d=d_{+}$and $q_{1} \neq 0$. The intersection takes place for $\left(D_{B}, T_{B}\right)=(0.77,0.40)$.

c. High density dynamical transition. At $\left(D_{\mathrm{th}}, T_{\mathrm{th}}\right) \simeq(0.876,0.379)$, the dynamical RFOT line and the spinodal SG line intersect. It can then be seen that for $D>D_{\text {th }}$, the dynamical RFOT line coincides with the spinodal line of the TFOPT, which means that the dynamical arrest in the $\mathrm{PM}^{+}$phase will take place as soon as phase separation occurs. From the thermodynamic point of view, we have coexistence between two paramagnets, as before. However, if we perform a quenching dynamics from the high density PM phase, a dynamical arrest into a metastable SG phase will take place.

\section{THE DYNAMICS}

We are now ready to derive the dynamical equations for the model. Let us first separate the disordered part of the Hamiltonian (4) from the deterministic one

$$
\begin{aligned}
H & =H_{0}+H_{J} \\
H_{0} & =(D-T \log 2) \sum_{i=1}^{N} \frac{\sigma_{i} \tau_{i}+1}{2} \\
H_{J} & =-\frac{1}{2^{p}} \sum_{i_{1}<i_{2}<\cdots<i_{p}} J_{i_{1} i_{2} \ldots i_{p}}\left(\sigma_{i 1}+\tau_{i 1}\right) \ldots\left(\sigma_{i p}+\tau_{i p}\right) .
\end{aligned}
$$

The relaxation dynamics is, then, governed by the $2 N$ Langevin equations

$$
\begin{gathered}
\dot{\sigma}_{i}=-\mu(t) \sigma_{i}(t)-\frac{\partial H_{0}}{\partial \sigma_{i}}-\frac{\partial H_{J}}{\partial \sigma_{i}}+\eta_{i}(t), \\
\dot{\tau}_{i}=-\nu(t) \tau_{i}(t)-\frac{\partial H_{0}}{\partial \tau_{i}}-\frac{\partial H_{J}}{\partial \tau_{i}}+\theta_{i}(t),
\end{gathered}
$$

where we assume the noise fields $\eta_{i}(t)$ and $\theta_{i}(t)$ to be delta-correlated:

$$
\begin{aligned}
\left\langle\eta_{i}(t) \eta_{j}\left(t^{\prime}\right)\right\rangle=\left\langle\theta_{i}(t) \theta_{j}\left(t^{\prime}\right)\right\rangle=\delta_{i j} D_{0}\left(t-t^{\prime}\right), \\
\left\langle\eta_{i}(t) \theta_{j}\left(t^{\prime}\right)\right\rangle=0
\end{aligned}
$$

with (taking the Boltzmann constant $k_{B}=1$ )

$$
D_{0}\left(t-t^{\prime}\right)=2 T \delta\left(t-t^{\prime}\right) .
$$

Following [53], we have inserted the Lagrange multipliers $\mu(t) \sigma_{i}(t)$ and $\nu(t) \tau_{i}(t)$ in order to enforce the spherical constraint.

The quantities we are interested in are the correlation functions and the response functions of the system. In this case, differently from the standard spherical p-spin model, we have two different types of degrees of freedom, and thus two possible external perturbing fields, one for each of them. As a result of this, we have four different correlation functions

$$
\begin{aligned}
C_{\sigma \sigma}\left(t, t^{\prime}\right) & =\frac{1}{N} \sum_{i=1}^{N} \overline{\left\langle\sigma_{i}(t) \sigma_{i}\left(t^{\prime}\right)\right\rangle}, \\
C_{\sigma \tau}\left(t, t^{\prime}\right) & =\frac{1}{N} \sum_{i=1}^{N} \overline{\left\langle\sigma_{i}(t) \tau_{i}\left(t^{\prime}\right)\right\rangle}, \\
C_{\tau \sigma}\left(t, t^{\prime}\right) & =\frac{1}{N} \sum_{i=1}^{N} \overline{\left\langle\tau_{i}(t) \sigma_{i}\left(t^{\prime}\right)\right\rangle}, \\
C_{\tau \tau}\left(t, t^{\prime}\right) & =\frac{1}{N} \sum_{i=1}^{N} \overline{\left\langle\tau_{i}(t) \tau_{i}\left(t^{\prime}\right)\right\rangle},
\end{aligned}
$$


and four response functions

$$
\begin{aligned}
& R_{\sigma \sigma}\left(t, t^{\prime}\right)=\frac{1}{N} \sum_{i=1}^{N} \frac{\delta \overline{\left\langle\sigma_{i}(t)\right\rangle}}{\delta h_{i}\left(t^{\prime}\right)} \\
& R_{\sigma \tau}\left(t, t^{\prime}\right)=\frac{1}{N} \sum_{i=1}^{N} \frac{\delta \overline{\left\langle\tau_{i}(t)\right\rangle}}{\delta h_{i}\left(t^{\prime}\right)}, \\
& R_{\tau \sigma}\left(t, t^{\prime}\right)=\frac{1}{N} \sum_{i=1}^{N} \frac{\delta \overline{\left\langle\sigma_{i}(t)\right\rangle}}{\delta l_{i}\left(t^{\prime}\right)} \\
& R_{\tau \tau}\left(t, t^{\prime}\right)=\frac{1}{N} \sum_{i=1}^{N} \frac{\delta \overline{\left\langle\tau_{i}(t)\right\rangle}}{\delta l_{i}\left(t^{\prime}\right)}
\end{aligned}
$$

where $h_{i}(t)$ and $l_{i}(t)$ are time-dependent perturbing fields conjugated with the $\sigma_{i}$ and $\tau_{i}$ degrees of freedom, respectively.

Our aim is to use equations (15) to obtain selfconsistent dynamical equations for the functions above. We employ the generating functional method devised in [54] by Martin, Siggia and Rose, and already used for the $p$-spin model in [55] by Kirkpatrick and Thirumalai. The MSR approach consist essentially in defining a generating functional $Z\left[\boldsymbol{h}, \boldsymbol{l}, \hat{\boldsymbol{h}}, \hat{\boldsymbol{l}}, J_{i j}\right]$ [56] for the $2 N$ 1-dimensional random fields $\sigma_{i}(t)$ and $\tau_{i}(t)[57]$. The correlation and response functions can, then, be obtained by taking functional derivatives of $Z$ with respect to the external fields $\boldsymbol{h}(t), \boldsymbol{l}(t), \hat{\boldsymbol{h}}(t)$ and $\hat{\boldsymbol{l}}(t)$, as in an usual field theory.

We have emphasized the fact that the generating functional still depends on the quenched random couplings $J_{i j}$, and so does every quantity generated by it; so, in principle, we would have to average them over the disorder in order to obtain the correlation and response functions we want. However, as remarked by De Dominicis in [58], since the generating functional in absence of external currents is by definition normalized to one

$$
Z\left[0,0,0,0, J_{i j}\right]=1,
$$

it is independent from the variables of the system, and so it can be averaged over the disorder directly. This is in contrast with the static partition function for a system with quenched disorder, which is not self-averaging. As a result of this, the use of replica theory is not needed in the dynamical framework; this fact constitutes the main advantage of the dynamical approach over the static one.

Performing the average over the disorder leads to a decoupling of the lattice sites and a coupling of the configurations of the system at different times, as it happens for the $p$-spin model in [55]. This is to be conceptually compared to the decoupling of the sites in the static approach with replicas, yielding a coupling between different replicas [59]. It is then possible, using saddle point methods, to write an effective generating functional which yields two single site dynamic equations valid for every degree of freedom in the lattice. The details of the derivation of the dynamics can be found in the appendix. We report here the site-independent dynamical equations

$$
\begin{aligned}
\dot{\sigma}= & -\mu \sigma(t)-\frac{(D-T \log 2)}{2} \tau(t) \\
& +K_{p}(p-1) \int d t^{\prime} \mathcal{R}\left(t, t^{\prime}\right) \mathcal{C}\left(t, t^{\prime}\right)^{p-2}\left(\sigma\left(t^{\prime}\right)+\tau\left(t^{\prime}\right)\right) \\
& +\xi(t), \\
\dot{\tau}_{i}= & -\nu \tau(t)-\frac{(D-T \log 2)}{2} \sigma(t) \\
& +K_{p}(p-1) \int d t^{\prime} \mathcal{R}\left(t, t^{\prime}\right) \mathcal{C}^{p-2}\left(t, t^{\prime}\right)\left(\sigma\left(t^{\prime}\right)+\tau\left(t^{\prime}\right)\right) \\
& +\zeta(t),
\end{aligned}
$$

where the correlation matrix for the noise terms $\xi(t)$ and $\zeta(t)$ has been renormalized in the following way

$$
\begin{aligned}
\left\langle\xi_{i}(t) \xi_{j}\left(t^{\prime}\right)\right\rangle & \left.=\delta_{i j} K_{p} \mathcal{C}\left(t, t^{\prime}\right)^{p-1}+\delta_{i j} 2 T \delta\left(t-t^{\prime}\right)\right), \\
& =\left\langle\zeta_{i}(t) \zeta_{j}\left(t^{\prime}\right)\right\rangle \\
\left\langle\xi_{i}(t) \zeta_{j}\left(t^{\prime}\right)\right\rangle & =\delta_{i j} K_{p} \mathcal{C}\left(t, t^{\prime}\right)^{p-1} .
\end{aligned}
$$

We have also defined the constant

$$
K_{p} \equiv \frac{J^{2} p}{2^{2 p+1}}
$$

and the two functions

$$
\begin{aligned}
\mathcal{C} & \equiv C_{\sigma \sigma}+C_{\sigma \tau}+C_{\tau \sigma}+C_{\tau \tau}, \\
\mathcal{R} & \equiv R_{\sigma \sigma}+R_{\sigma \tau}+R_{\tau \sigma}+R_{\tau \tau} .
\end{aligned}
$$

The equations for the correlation and response function can then be easily obtained, as reported in appendix A.

\section{A. Symmetries, equilibrium and ergodicity}

In appendix A, we derive eight coupled differential equations for eight different unknown functions. We now specify them to the particular problem we want to study, i.e. identifying dynamical arrest. In order to to this, we can restrict ourselves to an equilibrium (i.e. starting from an equilibrium initial condition) and ergodic dynamics. This implies time-translational invariance (TTI) of the correlators

$$
C\left(t, t^{\prime}\right)=C\left(t-t^{\prime}\right)
$$

and that the fluctuation-dissipation theorem (FDT) holds

$$
R(t)=-\theta(t) \frac{1}{T} \frac{d C(t)}{d t},
$$

where $R$ and $C$ denote any correlation and response function couple in the system, respectively, and $\theta(t)$ is the Heaviside step function. These assumptions are valid in the high temperature PM phase, where ergodicity is not 
broken, but they are generally false when the system is cooled below the dynamical transition temperature $T_{d}$, where a transition to a $S G$ phase with broken ergodicity takes place. Second, we notice that both the model Hamiltonian (4) and the effective generating functional (A9) are symmetric with respect to a $\boldsymbol{\sigma} \leftrightarrow \boldsymbol{\tau}$ switch

$$
H(\boldsymbol{\sigma} \rightarrow \boldsymbol{\tau}, \boldsymbol{\tau} \rightarrow \boldsymbol{\sigma})=H(\boldsymbol{\sigma}, \boldsymbol{\tau}) .
$$

This means that the $\boldsymbol{\sigma}$ and $\boldsymbol{\tau}$ evolve in the same statistical ensemble, which, in turn, implies that the correlation functions obey the relations

$$
C_{\sigma \sigma}\left(t, t^{\prime}\right)=C_{\tau \tau}\left(t, t^{\prime}\right), \quad C_{\sigma \tau}\left(t, t^{\prime}\right)=C_{\tau \sigma}\left(t, t^{\prime}\right),
$$

that can then be extended to the response functions by exploiting the FDT

$$
\begin{aligned}
& R_{\sigma \sigma}=-\theta(t) \frac{1}{T} \frac{d C_{\sigma \sigma}}{d t}=-\theta(t) \frac{1}{T} \frac{d C_{\tau \tau}}{d t}=R_{\tau \tau} \\
& R_{\sigma \tau}=-\theta(t) \frac{1}{T} \frac{d C_{\sigma \tau}}{d t}=-\theta(t) \frac{1}{T} \frac{d C_{\tau \sigma}}{d t}=R_{\tau \sigma} .
\end{aligned}
$$

Once that these relations are established, we can see that only the two correlation functions $C_{\sigma \sigma}$ and $C_{\sigma \tau}$ are needed to completely describe the dynamics of the system. Thus, we can define the "total" correlation function of the system $C\left(t-t^{\prime}\right)$, by normalizing the $\mathcal{C}$ to one

$$
C\left(t-t^{\prime}\right)=\frac{\mathcal{C}\left(t-t^{\prime}\right)}{\mathcal{C}(0)}=\frac{2\left(C_{\sigma \sigma}\left(t-t^{\prime}\right)+C_{\sigma \tau}\left(t-t^{\prime}\right)\right)}{4 d(0)} .
$$

where we have used the spherical constraint

$$
\sum_{i=1}^{N}\left\langle\sigma_{i}(t) \sigma_{i}(t)\right\rangle=N \Longrightarrow C_{\sigma \sigma}(t, t)=1
$$

and the relation (3) between the $\sigma \tau$ product and the site occupation number

$$
\begin{aligned}
\frac{\sigma_{i}(t) \tau_{i}(t)+1}{2} & =n_{i}(t) \\
\Longrightarrow C_{\sigma \tau}(t, t)=C_{\tau \sigma}(t, t) & =2 d(t)-1 .
\end{aligned}
$$

Since we assume the dynamics to be at equilibrium, the density $d(t)$, being an one-time quantity, is a constant of motion, equal to its equilibrium value

$$
d(0)=d(t)=d(\infty) \quad \forall t .
$$

Using the equations for the $C_{\sigma \sigma}$ and $C_{\sigma \tau}$, we obtain the following integro-differential equation for the total correlation function $C(t)$

$$
\begin{aligned}
\dot{C}(t)= & \mathcal{A}(D, T, d) C(t) \\
& -\mathcal{B}(T, d) \int_{0}^{t} d u C^{p-1}(t-u) \dot{C}(u) \\
\mathcal{A}(D, T, d) \equiv & \left(D-T \log 2-\frac{J^{2} d^{p-1} p}{4 T}\right)(d-1)-T \\
\mathcal{B}(T, d) \equiv & \frac{J^{2} p d^{p-1}}{4 T}
\end{aligned}
$$

which is a closed equation, whose solution can be found once the values of the parameters $D, T$ and $d$ are known. It can be seen as a Mode Coupling schematic equation, and it is worth noticing that in the limit of the $d$ parameter (the fraction of filled-in sites) going to 1 one recovers standard MC equations. The density $d$ is assumed, at all times, to be equal to its equilibrium value, given by Eq. (12). As a result of this, in the region between spinodal lines, where phase coexistence occurs, the dynamics will have to be studied separately for each one of the two coexisting phases. In the high density limit case $d \rightarrow 1$, thermodynamically occurring for low $D$, the proper limit of Eq. (27) is considered in appendix A 3. The study of Eq. (27) will be one of the main focuses of this work.

An equation can be derived also for the difference $\Delta C$ between $C_{\sigma \sigma}$ and $C_{\sigma \tau}$, obtaining

$$
\frac{d(\Delta C(t))}{d t}=\left[(D-T \log 2) d-\frac{p}{4 T} d^{p}-T\right] \Delta C(t),
$$

which has the trivial solution

$$
\Delta C(t)=2(1-d) e^{A t}
$$

where the $A$ constant is defined as

$$
A \equiv(D-T \log 2) d-\frac{p}{4 T} d^{p}-T .
$$

If now we use Eq. (12) to eliminate $D$, we get

$$
A=-\frac{T}{2(1-d)}
$$

which is always negative for any value of $d$ and $T$. This means that the difference between the two correlators tends to zero for long enough times, and thus the dynamics of the system can be always solved using the $C(t)$ function only.

\section{B. Solving the dynamic equations}

In this section we report the results obtained by numerically solving equation (27) in various representative points of the phase diagram. Eq. (27) is an integrodifferential mode-coupling like equation [39], that can be solved using the standard algorithm intruced by Fuchs et al. [60] and extended in different ways, cf. e. g., Refs. [61-63]. We consider three meaningful cases to illustrate the varoius occuring regimes.

\section{1. $D=0:$ dynamic transition}

At $D=0$ and high $T$ the system yields a single paramagnetic phase (PM), which undergoes a dynamical transition with ergodicity breaking at $T_{d}(D=0)=0.4892$ [13]. In figure 2 we report the total correlation function of the system as the transition is approached from above. 


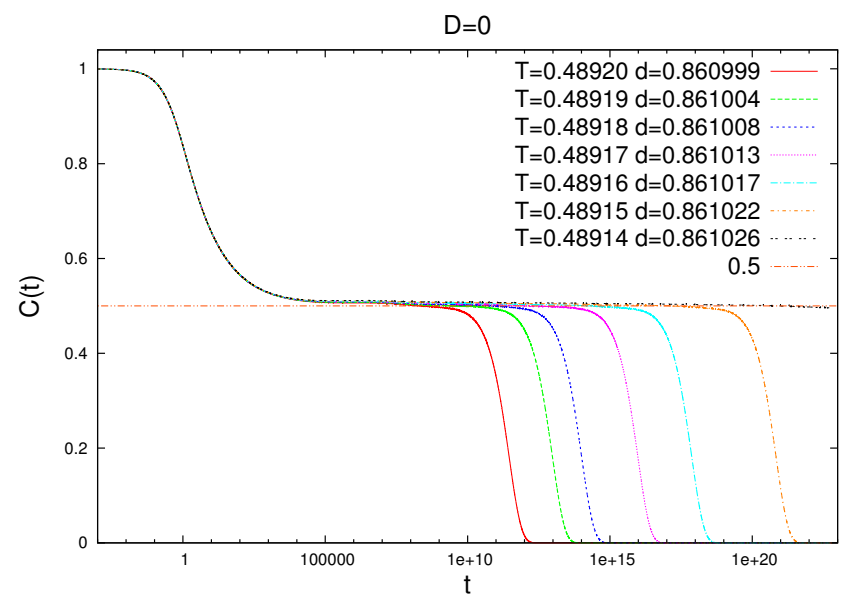

FIG. 2. The total correlation function $C(t)$ evaluated for various temperatures in the vicinity of the dynamical transition at $T_{d}(D=0)=0.4892$; for each temperature, the value of the equilibrium density $d$ of the system is reported. As the system is cooled, the function develops a plateau whose length increases rapidly with decreasing $T$, to become infinite at the dynamical transition line.

As we can see, our dynamic equation (27) yields the dynamic transition predicted in [13], and the correlator shows the typical phenomenology of a mode-coupling like dynamic arrest. The transition temperature $T_{d}$ corresponds up to a $O\left(10^{-4}\right)$ error with the predicted value 0.4892 and the height of the plateau $q_{d}$ (also called the non-ergodicity parameter $)$ is $(p-2) /(p-1)=0.5$, as expected.

\section{2. $D=D_{B}:$ phase coexistence}

For $D=D_{B}=0.77$, the situation is richer and more interesting; for this value of the crystal field, at low temperature the system undergoes phase coexistence, yielding two separate paramagnetic phases with high $\left(\mathrm{PM}^{+}\right)$ and low $\left(\mathrm{PM}^{-}\right)$density. As we anticipated, the dynamics of the system has to be solved separately for each one of these two phases. Their behavior turns out, actually, to be quite different, as only the high density $\mathrm{PM}+$ phase undergoes ergodicity breaking as the dynamical line is crossed. In figure 3 we plot the resulting correlators as the system enters the phase coexistence zone, and the high density phase undergoes the dynamic transition.

We see that, again, the expected phenomenology is reproduced by our equation. At $T=0.42586$ only a single paramagnetic phase is present, but for $T=0.41586$, two different paramagnetic phases separate; at $T=$ $0.40586 \simeq T_{d}(D=0.77)$, the high density phase correlator shows the typical plateau as the dynamic transition is approached, and as the cooling continues, the high density $\mathrm{PM}^{+}$phase eventually undergoes a dynamical arrest,

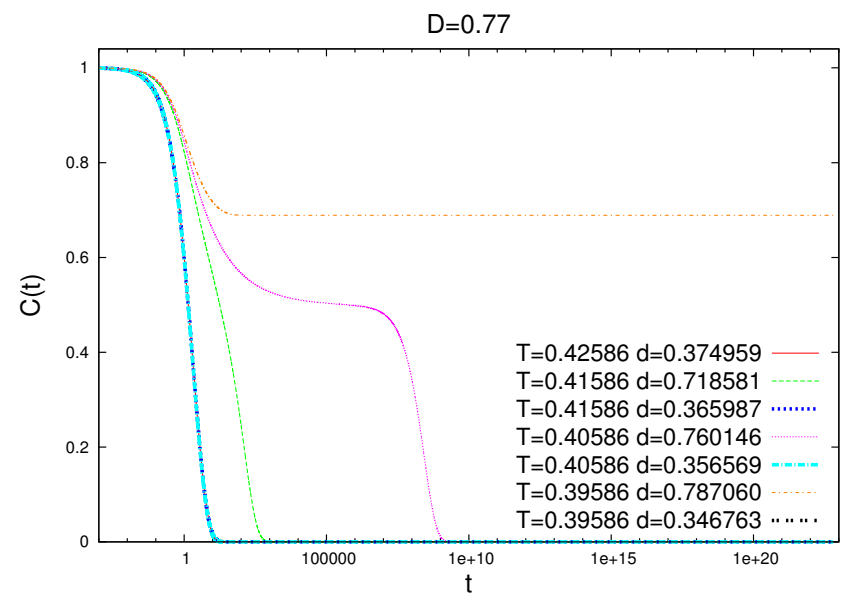

FIG. 3. The total correlation function $C(t)$ evaluated for various temperatures as the system enters the phase coexistence zone and the high density phase undergoes dynamical arrest. Here $T_{d}(D=0.77) \simeq 0.40586$. In the phase coexistence zone, the $\mathrm{PM}+$ correlator is plotted with a thin line, while the PMwith a thick one. As we can see, the $\mathrm{PM}+$ makes a transition to an arrested SG phase while the $\mathrm{PM}$ - remains paramagnetic.

while the low density paramagnet $\mathrm{PM}^{-}$stays unchanged until the first order phase transition takes place to the thermodynamic 1RSB-stable glass phase.

\section{3. $D>D_{t h}$ : anomalous dynamical arrest}

Up to the prevuoius case, our dynamic analysis confirms the results of the static one [13]. However, as we anticipated in section $\mathrm{I}$, for $D>D_{t h}$, our dynamical equation yield dynamic arrest at finite temperature when starting from the $\mathrm{PM}^{+}$phase, an effect that was not identified in the static analysis, where the thermodynamically dominant phase is low density $\mathrm{PM}^{-}$. We shall now report the results obtained by solving equation (27), leaving the study and discussion of the new arrested phase for the next section.

In order to show the onset of this new transition, we choose $D=1>D_{t h}, T=0.35$ and we cool the system starting from a high density initial condition crossing the spinodal line during the procedure. The $\mathrm{PM}^{+}$spinodal line, for these chemical potential values, turns to be a dynamical arrest transition line, as shown in figure 4 . We can observe that, as soon as the two PM phases separate, the high density phase is already an arrested SG phase, with a nonzero overlap, while the low density phase shows no sign of dynamical arrest. This is at difference with respect to the static results of Ref. [13], where the high density phase is supposed to be still paramagnetic for $T=0.34$.

What is puzzling about this result is the fact that the 
high density phase is already deep into the $\mathrm{SG}$ when the separation occurs: for $T=0.34$ we have $q_{d} \approx 0.82$, which is already much higher than the $(p-2) /(p-1)=0.5$ that we would expect for a system which approaches from above an usual dynamical arrest. This means that if the high density phase existed even above the spinodal line, its dynamic transition temperature would be actually much higher than $T=0.34$; however, this effect is not visible since only the low density $\mathrm{PM}^{-}$phase exists in that region. If we follow the same cooling procedure for $D=2$ (i.e., we cross the spinodal line at $D=2$ during the cooling), the results are not very different, and the high density arrested phase is still present. In this case, the value of the overlap at the separation line is even higher, with $q_{d} \approx 0.97$ for $T=0.2$.

Solving equation (27) for higher $D$ does not change the general situation, so we will not report any results for higher values. The point is that, since the spinodal line is an asymptote of the $D$ axis (as we have mentioned in section II), then for arbitrarily large $D$, a high density phase exists at $T>0$. According to both the marginality condition for the statics, and the dynamic results presented so far, this phase presents a dynamical arrest into a SG phase with nonzero overlap, that can be realized by selecting atypically dense initial conditions at those temperature and chemical potential.

\section{COMPLEXITY AND FREE ENERGY}

Since the system undergoes a dynamical arrest, we would expect the RFOT phenomenology which holds in the other regions of the phase diagram to be present in this case as well, inside the high $d$ minimum which corresponds to the $\mathrm{PM}+$ phase (the low density paramagnet is completely orthogonal to our discussion). In summary, we expect the metastable states which trap the dynamics (and maximize the complexity) to have a higher in-state free-energy than the one of the paramagnetic, ergodic state. We might also expect a complexity $\Sigma(f)$ to be strictly positive for every $f$ up to a static temperature $T_{s}(D)$ where states with null complexity are born and a static transition takes place. We computed both quantities using replica theory as in Ref. [13] and report the corresponding curves in figures 5 and 6 . We see that the usual RFOT picture does not hold for all values of $D$ along the new dynamical arrest line. Indeed, it holds only for $D<D_{i n v}$ where $D_{i n v} \simeq 0.9062$, which in fact corresponds to the point where the static line touches the new dynamical arrest line. In this region, the complexity of the states is positive for every $f$ (as one can see in the upper panel of figure 6) and the free energy of the $m=1$ states which trap the dynamics is higher than the paramagnetic one. On lowering $T$ at constant $D$, the complexity touches zero (corresponding to the condition Eq. (9)) and a static transition to a SG phase takes place as usual.

This picture does not hold anymore for $D \geq D_{i n v} \simeq$
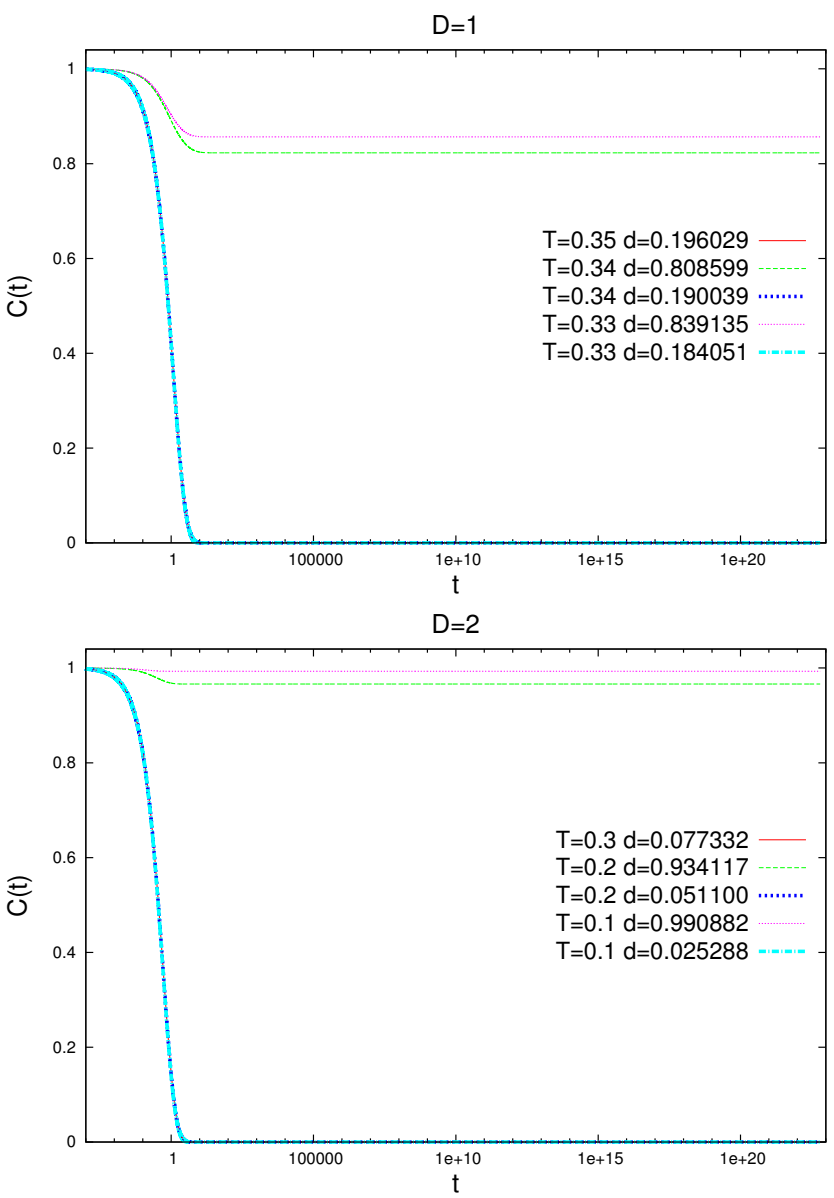

FIG. 4. The total correlation function $C(t)$ evaluated for various temperatures as the system enters the phase coexistence zone and the anomalous dynamic transition takes place. The presence of this dynamic transition is a novelty with respect to the results of [13].

0.9062. For $D=D_{i n v}$, the maximum complexity condition (dynamical arrest line) and the zero complexity condition (static line) coincide, which means that the complexity is zero (i.e., the number of all states becomes subexponential) and the free energy of the trapping states becomes equal to the paramagnetic $(\mathrm{PM}+)$, cf. figure 5 . This is alike to the occurrence of the static Kauzmann transition but in this case both SG and $\mathrm{PM}^{+}$ phases are metastable and dynamically occurring only for initial conditions with a density atypically high for these chemical potential $D$ values. When $D$ is increased along the high density arrest line, the free energy of the trapping states becomes lower than the paramagnetic one and continues to decrease indefinitely, while the complexity becomes more and more negative as reported in figure 6 . If one looks at the phase diagram 1 , it is possible to see that there is a range of $D$ such that, on cooling, the static line is met before the dynamic one [13]. This would mean that along this path in the parame- 


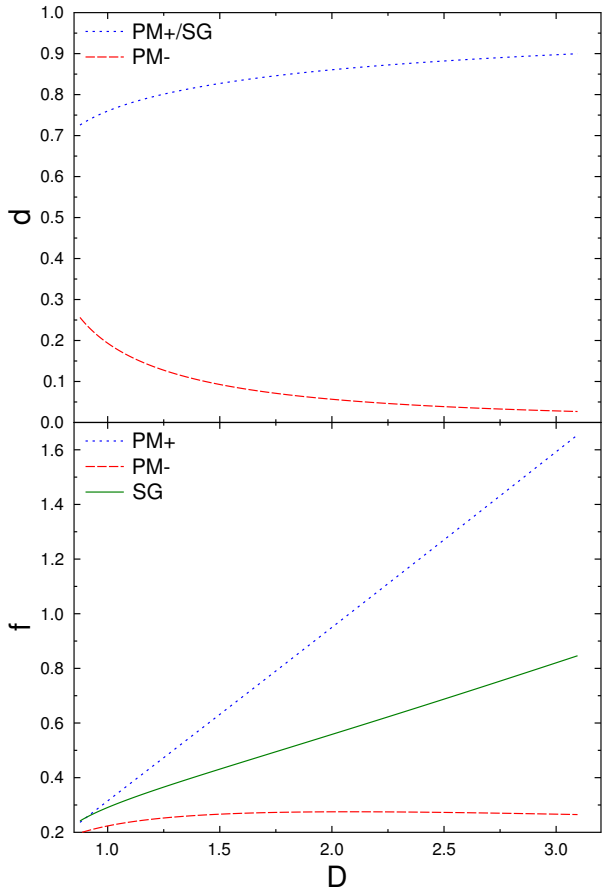

FIG. 5. The state free energy of the low density paramagnet (PM-), the high density paramagnet $(\mathrm{PM}+)$, and of the spin glass states which trap the dynamics (SG), evaluated along the high density dynamical arrest line for $D>D_{t h}=0.876$. We also report the densities of the two paramagnets. We see that the free energy $f$ of the trapping states becomes equal to the one of the paramagnet for $D=D_{\text {inv }} \simeq 0.9062$

ter space the states with low free energy and complexity arise before the ones with maximum complexity and free energy, only eventually appearing on the maximum complexity line (that, for $D<D_{t h}$, is the dynamic line): the complexity of the glassy metastable states becomes again non-zero crossing the static line on cooling and reaches its maximum at the line formerly denoted as dynamic line. However, this is true for $D>D_{t h} \simeq 0.876$ up until $D \sim 1.6$. From that point on, the static line touches the $T=0$ axis and the complexity stays negative for every value of $D, T$. In summary, using both the dynamical equations and the replica approach, we find an anomalous, complexity free, dynamical transition, that occurs if the system is initially prepared at values of the density corresponding to a paramagnetic metastable state at high density. This state always coexists with a more probable and thermodynamically dominant low density paramagnetic state. It is the latter which, at lower $T$ and/or lower $D$, undergoes a first order phase transition to a spin-glass phase.
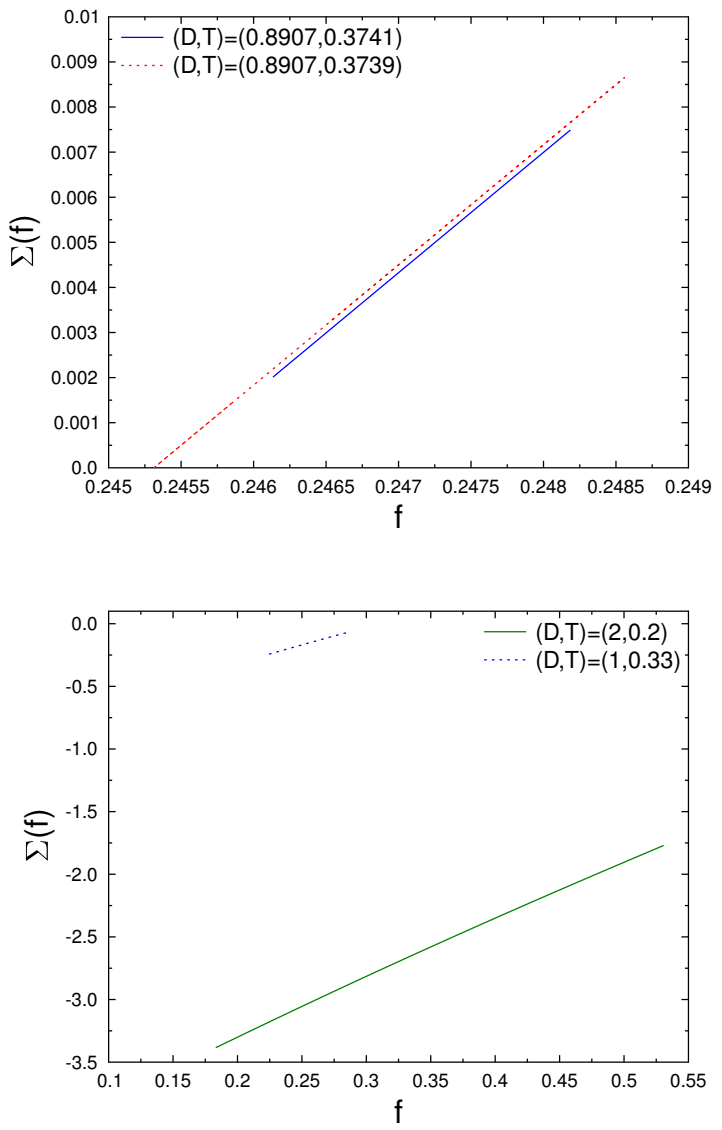

FIG. 6. The complexity of the states which trap the dynamics, below the high density dynamical arrest line. Upper Panel: complexity for two points with $D<D_{\text {inv }} \simeq 0.9062$, with $T_{s}(D)<T<T_{d}(D)=\ldots$ (full line) and $T=T_{s}(D)=\ldots$ (dotted line), respectively. Lower panel: complexity for two representative points with $D>D_{i n v}$.

\section{CONCLUSIONS}

In this work, we have studied, both dynamically and statically, a disordered model which shows both an RFOT-like phenomenology (dynamical arrest, complexity, Kauzmann transition, etc.), and an "ordinary" first order phase transition with latent heat and phase coexistence. We have derived and solved the equations for the equilibrium dynamics of the model and completed the static replica-based study of [13] with novel results. In doing so, we have noticed the presence, in a certain region of the phase diagram, of a RFOT-like dynamical arrest line, which however shows a non-positive complexity and does not work as a precursor for a Kauzmann-like static transition, in contrast with the usual phenomenology expected in RFOT models.

The picture we propose to explain this is the following. Our model has two order parameters, the density $d$ and the 1RSB self-overlap $q_{1}$ (termed $q$ from now on). One 
can imagine to construct a potential function of $d$ and $q$, by plotting the paramagnetic free energy as a function of $d$, and then performing a Franz-Parisi like [50] construction along the $q$ axis, for every $d$. For $D<D_{B}=0.77$ the transition scenario is qualitatively alike to the random first order transition one.

For chemical potential values in the interval $D_{B}<$ $D<D_{t h}$, instead, the scenario changes. For high $T$ only one minimum is present but, on cooling, the spinodal line of a second paramagnetic phase, at higher density, is crossed: a second, metastable minimum with $q=0$ and density $d=d^{+}$is formed, termed $P M^{+}$. Further lowering the temperature the dynamical transition line is crossed. There, a metastable phase with $q \neq 0$ and $d=d^{+}$arises from the $\mathrm{PM}^{+}$solution, with a higher free energy. This phase consists of many equivalent states and corresponds to the arrested glassy phase. The difference in free energy between the SG metastable minima and the free energy of the $\mathrm{PM}^{+}$minimum is equal to the complexity counting the log of the number of SG metastable states. If $T$ is lowered further, we cross the static transition line, where the $q \neq 0$ minimum has the same height as the paramagnetic one (null complexity) and thus it becomes stable: we have a static transition in a spin-glass phase.

To summarize, we have a three-step process on cooling for $D \in\left[D_{B}, D_{\mathrm{th}}\right]$ :

1. A secondary $\mathrm{PM}^{+}$minimum with $d=d+$ and $q=0$ is formed (the stable phase is the low density $\mathrm{PM}^{-}$).

2. A SG minimum with $d=d+, q \neq 0$ is formed, arising from $\mathrm{PM}^{+}$.

3. The SG becomes stable with respect to the low density paramagnet $\mathrm{PM}^{-}$and a static transition takes place.

This scenario almost corresponds the usual RFOT phenomenology. The only difference is that there are two paramagnetic phases, one stable $\left(\mathrm{PM}^{-}\right)$and one metastable at higher density, $\mathrm{PM}^{+}$, and that the glassy metastable states at the threshold free energy arise inside the $P M^{+}$.

When $D$ is raised beyond $D_{\mathrm{th}}$, the order of these steps above is changed. Two apart scenarios appear. For $D_{\text {th }}<D<D_{\text {inv }}$, steps 1 and 2 exchange. Fixing $T$ slightly above $T_{\mathrm{sp}}(D), d=d^{+}$and looking at the potential only in the direction of $q$, the $q \neq 0$ minimum will have already formed. However, this has no effect on the thermodynamics since $d=d^{+}$does not yet correspond to a minimum on the $d$ axis. For that to happen, $T$ must be lowered to cross the $\mathrm{PM}^{+}$spinodal. At lower $T$ the process goes on as previously.

For $D>D_{\text {inv }}$, step 1 becomes the last to happen: again, the system dynamics is arrested as soon as the $\mathrm{PM}^{+}$spinodal is reached, but the $\mathrm{SG}$ minimum along $q$ not only is formed before the $\mathrm{PM}^{+}$has a chance to appear, but it has even become stable with respect to the $\mathrm{PM}^{+}$. We stress the fact that this unusual behavior is possible only because the system has two order parameters, differently from usual RFOT models whose behavior is governed only by $q$. The fact that the minimum in $q$ is already formed for $D>D_{\text {th }}$ is, indeed, confirmed by the fact that the naive marginality condition $d^{2} F / d q^{2}=0$, used in Ref. [13], completely misses this new arrest line: in the direction of $q$ the minimum is already formed and the curvature is positive. The whole Hessian matrix of the replicated free energy with respect to $q$ and $d$ (evaluated at $m=1$ ) must be used to detect the new line, as reported in Sec. II A. This picture is corroborated by the fact that this phenomenology is found in the $\mathrm{PM}^{+}$phase, whose density goes up and approaches 1 as $D$ increases, as reported in the upper panel of figure 5: this means that the $\mathrm{PM}^{+}$phase of our model becomes more and more similar to the usual $p$-spin spherical model (PSM) [30], and the dynamical equations behave accordingly as explained in appendix A 3. However, we also stress that such density values are thermodynamically extremely unlikely to occur for these large values of the chemical potential $D$.

\section{ACKNOWLEDGEMENTS}

The research leading to these results has received funding from the Italian Ministry of Education, University and Research under the Basic Research Investigation Fund (FIRB/2008) program/CINECA grant code RBFR08M3P4 and under the PRIN2010 program, grant code 2010HXAW77-008, and from the European Research Council, under the European Union's Seventh Framework Programme FP7/2007-2013/ from the People Programme (Marie Curie Actions) under REA grant agreement n 290038, NETADIS project, and ERC grant agreement n 247328, CryPheRaSy project. U.F. thanks A. Destexhe for hosting at the European Institute for Theoretical Neuroscience. 
Appendix A: Derivation of the dynamical equations for the p-spin Blume-Capel spherical model

In this appendix we provide the step-by-step derivation of the dynamical equations studied in this paper.

\section{Computation of the effective generating functional}

We write down the full expression for the MSR generating functional

$$
\begin{aligned}
Z\left[\boldsymbol{h}, \boldsymbol{l}, \hat{\boldsymbol{h}}, \hat{\boldsymbol{l}}, J_{i j}\right] & =\int \mathcal{D} \sigma \mathcal{D} \hat{\sigma} \mathcal{D} \tau \mathcal{D} \hat{\tau} \times \exp \left[-\frac{1}{2} \int d t d t^{\prime} \hat{\boldsymbol{\sigma}}(t) \cdot \hat{\boldsymbol{\sigma}}(t) D_{0}\left(t-t^{\prime}\right)+\hat{\boldsymbol{\tau}}(t) \cdot \hat{\boldsymbol{\tau}}\left(t^{\prime}\right) D_{0}\left(t-t^{\prime}\right)\right] \\
& \times \exp \left[\int d t i\left(\hat{\boldsymbol{\sigma}}(t) \cdot\left(\dot{\boldsymbol{\sigma}}(t)+\mu(t) \boldsymbol{\sigma}(t)+\boldsymbol{\partial}_{\boldsymbol{\sigma}} \boldsymbol{H}_{\mathbf{0}}(t)\right)+\hat{\boldsymbol{\tau}} \cdot\left(\dot{\boldsymbol{\tau}}(t)+\nu(t) \boldsymbol{\tau}(t)+\boldsymbol{\partial}_{\boldsymbol{\tau}} \boldsymbol{H}_{\mathbf{0}}(t)\right)\right)\right] \\
& \left.\times \exp \left[\int d t i\left(\hat{\boldsymbol{\sigma}}(t) \cdot \boldsymbol{\partial}_{\boldsymbol{\sigma}} \boldsymbol{H}_{\boldsymbol{J}}(t)\right)+\hat{\boldsymbol{\tau}}(t) \cdot \boldsymbol{\partial}_{\boldsymbol{\tau}} \boldsymbol{H}_{\boldsymbol{J}}(t)\right)\right], \\
& \times \exp \left[-\int d t i(\boldsymbol{h}(t) \cdot \hat{\boldsymbol{\sigma}}(t)+\hat{\boldsymbol{h}}(t) \cdot \boldsymbol{\sigma}(t)+\boldsymbol{l}(t) \cdot \hat{\boldsymbol{\tau}}(t)+\hat{\boldsymbol{l}}(t) \cdot \boldsymbol{\tau}(t))\right]
\end{aligned}
$$

where $\mathcal{D}$ denotes a functional integration measure over all lattice sites; for example

$$
\mathcal{D} \sigma \equiv \prod_{i=1}^{N} \delta \sigma(t)
$$

The first step is to perform the average over the disorder of expression (A1) for the generating functional; since the disorder is contained only in the $H_{J}$ part of the hamiltonian, we have to perform the average only on the third line of the (A1). Thus we have to compute the integral

$$
\begin{aligned}
&\left.\exp \left[\int d t i\left(\hat{\boldsymbol{\sigma}}(t) \cdot \boldsymbol{\partial}_{\boldsymbol{\sigma}} \boldsymbol{H}_{\boldsymbol{J}}(t)\right)+\hat{\boldsymbol{\tau}}(t) \cdot \boldsymbol{\partial}_{\boldsymbol{\tau}} \boldsymbol{H}_{\boldsymbol{J}}(t)\right)\right]= \\
& \prod_{i_{1} i_{2} \ldots i_{p}} \int d J_{i_{1} i_{2} \ldots i_{p}} \exp \left\{-\frac{J_{i_{1} i_{2} \ldots i_{p}}^{2} N^{p-1}}{J^{2} p !^{2}}-\frac{J_{i_{1} i_{2} \ldots i_{p}}}{2^{p} p !} \int d t\left[i\left(\hat{\sigma}_{i_{1}}+\hat{\tau}_{i_{1}}\right)\left(\sigma_{i_{2}}+\tau_{i_{2}}\right) \ldots\left(\sigma_{i_{p}}+\tau_{i_{p}}\right)\right.\right. \\
&\left.\left.+i\left(\sigma_{i_{1}}+\tau_{i_{1}}\right)\left(\hat{\sigma}_{i_{2}}+\hat{\tau}_{i_{2}}\right) \ldots\left(\sigma_{i_{p}}+\tau_{i_{p}}\right)+\cdots+\left(\sigma_{i_{1}}+\tau_{i_{1}}\right)\left(\sigma_{i_{2}}+\tau_{i_{2}}\right) \ldots i\left(\hat{\sigma}_{i_{p}}+\hat{\tau}_{i_{p}}\right)\right]\right\}
\end{aligned}
$$

where we have symmetrized the $(\hat{\sigma}+\hat{\tau})_{i_{1}}(\sigma+\tau)_{i_{2}} \ldots(\sigma+\tau)_{i_{p}}$ couplings; performing the gaussian integration yields

$$
\begin{aligned}
&\left.\exp \left[\int d t i\left(\hat{\boldsymbol{\sigma}}(t) \cdot \boldsymbol{\partial}_{\boldsymbol{\sigma}} \boldsymbol{H}_{\boldsymbol{J}}(t)\right)+\hat{\boldsymbol{\tau}}(t) \cdot \boldsymbol{\partial}_{\boldsymbol{\tau}} \boldsymbol{H}_{\boldsymbol{J}}(t)\right)\right]= \\
& \frac{J^{2}}{2^{2 p+2} N^{p-1}} \int d t d t^{\prime}\left\{p\left[i(\hat{\boldsymbol{\sigma}}+\hat{\boldsymbol{\tau}})_{t} \cdot i(\hat{\boldsymbol{\sigma}}+\hat{\boldsymbol{\tau}})_{t^{\prime}}\right]\left[(\boldsymbol{\sigma}+\boldsymbol{\tau})_{t} \cdot(\boldsymbol{\sigma}+\boldsymbol{\tau})\right]_{t^{\prime}}^{p-1}+\right. \\
&\left.p(p-1)\left[i(\hat{\boldsymbol{\sigma}}+\hat{\boldsymbol{\tau}})_{t} \cdot(\boldsymbol{\sigma}+\boldsymbol{\tau})_{t^{\prime}}\right]\left[(\boldsymbol{\sigma}+\boldsymbol{\tau})_{t} \cdot i(\hat{\boldsymbol{\sigma}}+\hat{\boldsymbol{\tau}})_{t^{\prime}}\right]\left[(\boldsymbol{\sigma}+\boldsymbol{\tau})_{t} \cdot(\boldsymbol{\sigma}+\boldsymbol{\tau})_{t^{\prime}}\right]^{p-2}\right\} .
\end{aligned}
$$

As we anticipated in section III, the average over the disorder has decoupled the lattice sites, at the price of generating a coupling between configurations of the system at different times.
This dynamical coupling is conceptually similar to the coupling between replicas that occurs in the static treatment of the $p$-spin model [30, 52]; so, following [55], we define the dynamical overlaps between auxiliary MSR 
fields,

$$
\begin{aligned}
Q_{1}\left(t, t^{\prime}\right) & =\frac{1}{N} \sum_{i=1}^{N} i \hat{\sigma}_{i}(t) i \hat{\sigma}_{i}\left(t^{\prime}\right), \\
Q_{2}\left(t, t^{\prime}\right) & =\frac{1}{N} \sum_{i=1}^{N} i \hat{\sigma}_{i}(t) i \hat{\tau}_{i}\left(t^{\prime}\right), \\
Q_{3}\left(t, t^{\prime}\right) & =\frac{1}{N} \sum_{i=1}^{N} i \hat{\tau}_{i}(t) i \hat{\sigma}_{i}\left(t^{\prime}\right), \\
Q_{4}\left(t, t^{\prime}\right) & =\frac{1}{N} \sum_{i=1}^{N} i \hat{\tau}_{i}(t) i \hat{\tau}_{i}\left(t^{\prime}\right),
\end{aligned}
$$

between dynamical fields,

$$
\begin{aligned}
Q_{5}\left(t, t^{\prime}\right) & =\frac{1}{N} \sum_{i=1}^{N} \sigma_{i}(t) \sigma_{i}\left(t^{\prime}\right), \\
Q_{6}\left(t, t^{\prime}\right) & =\frac{1}{N} \sum_{i=1}^{N} \sigma_{i}(t) \tau_{i}\left(t^{\prime}\right), \\
Q_{7}\left(t, t^{\prime}\right) & =\frac{1}{N} \sum_{i=1}^{N} \tau_{i}(t) \sigma_{i}\left(t^{\prime}\right), \\
Q_{8}\left(t, t^{\prime}\right) & =\frac{1}{N} \sum_{i=1}^{N} \tau_{i}(t) \tau_{i}\left(t^{\prime}\right),
\end{aligned}
$$

and between auxiliary and dynamical fields

$$
\begin{aligned}
& Q_{9}\left(t, t^{\prime}\right)=\frac{1}{N} \sum_{i=1}^{N} i \hat{\sigma}_{i}(t) \sigma_{i}\left(t^{\prime}\right)=Q_{13}\left(t^{\prime}, t\right) \\
& Q_{10}\left(t, t^{\prime}\right)=\frac{1}{N} \sum_{i=1}^{N} i \hat{\sigma}_{i}(t) \tau_{i}\left(t^{\prime}\right)=Q_{14}\left(t^{\prime}, t\right) \\
& Q_{11}\left(t, t^{\prime}\right)=\frac{1}{N} \sum_{i=1}^{N} i \hat{\tau}_{i}(t) \sigma_{i}\left(t^{\prime}\right)=Q_{15}\left(t^{\prime}, t\right) \\
& Q_{12}\left(t, t^{\prime}\right)=\frac{1}{N} \sum_{i=1}^{N} i \hat{\tau}_{i}(t) \tau_{i}\left(t^{\prime}\right)=Q_{16}\left(t^{\prime}, t\right)
\end{aligned}
$$

These functions can be incorporated in the (A3) by inserting 1-factors in the form of functional Dirac deltas expressed in exponential form, for example

$$
\begin{aligned}
1=\int \delta Q_{1} \delta \lambda_{1} \exp & {\left[\int d t d t^{\prime} i \lambda_{1}\left(t, t^{\prime}\right)\right.} \\
& \left.\times\left(N Q_{1}\left(t, t^{\prime}\right)-\sum_{i=1}^{N} i \hat{\sigma}_{k}(t) i \hat{\sigma}_{k}\left(t^{\prime}\right)\right)\right]
\end{aligned}
$$

the (A3) then assumes the form

$$
\begin{aligned}
& \int \delta^{16} Q \delta^{16} \lambda \exp \left\{\int d t d t^{\prime} N\left[\sum_{\mu=1}^{16} i \lambda_{\mu} Q_{\mu}\right]\right\} \\
& \times \exp \left\{\frac { N J ^ { 2 } } { 2 ^ { 2 p + 2 } } \int d t d t ^ { \prime } \left[p\left(Q_{1}+Q_{2}+Q_{3}+Q_{4}\right)\left(Q_{5}+Q_{6}+Q_{7}+Q_{8}\right)^{p-1}+\right.\right. \\
& \left.\left.p(p-1)\left(Q_{9}+Q_{10}+Q_{11}+Q_{12}\right)\left(Q_{13}+Q_{14}+Q_{15}+Q_{16}\right)\left(Q_{5}+Q_{6}+Q_{7}+Q_{8}\right)^{p-2}\right]\right\} \\
& \times \exp \left\{-i \int d t d t^{\prime}\left[\lambda_{1} i \hat{\boldsymbol{\sigma}} \cdot i \hat{\boldsymbol{\sigma}}+\lambda_{2} i \hat{\boldsymbol{\sigma}} \cdot i \hat{\boldsymbol{\tau}}+\cdots+\lambda_{16} \boldsymbol{\tau} \cdot i \hat{\boldsymbol{\tau}}\right]\right\} .
\end{aligned}
$$

This expression contains an exponent proportional to the system size $N$, which in the thermodynamic limit allows us to perform the functional integrals over the $Q \mathrm{~s}$ and $\lambda \mathrm{s}$ using the saddle-point method. The saddle-point equa-

tions read

$$
\begin{aligned}
i \lambda_{1} \ldots i \lambda_{4}= & -\frac{J^{2} p}{2^{2 p+2}}\left(Q_{5}+Q_{6}+Q_{7}+Q_{8}\right)^{p-1} \\
i \lambda_{5} \ldots i \lambda_{8}= & -\frac{J^{2} p(p-1)}{2^{2 p+2}}\left(Q_{1}+Q_{2}+Q_{3}+Q_{4}\right) \\
& \times\left(Q_{5}+Q_{6}+Q_{7}+Q_{8}\right)^{p-2} \\
& -\frac{J^{2} p(p-1)(p-2)}{2^{2 p+2}}\left(Q_{9}+Q_{10}+Q_{11}+Q_{12}\right) \\
& \times\left(Q_{13}+Q_{14}+Q_{15}+Q_{16}\right) \\
& \left(Q_{5}+Q_{6}+Q_{7}+Q_{8}\right)^{p-3}
\end{aligned}
$$




$$
\begin{aligned}
i \lambda_{9} \ldots i \lambda_{12}= & -\frac{J^{2} p(p-1)}{2^{2 p+2}}\left(Q_{13}+Q_{14}+Q_{15}+Q_{16}\right) \\
& \times\left(Q_{5}+Q_{6}+Q_{7}+Q_{8}\right)^{p-2}, \\
i \lambda_{12} \ldots i \lambda_{16}= & -\frac{J^{2} p(p-1)}{2^{2 p+2}}\left(Q_{9}+Q_{10}+Q_{11}+Q_{12}\right) \\
& \times\left(Q_{5}+Q_{6}+Q_{7}+Q_{8}\right)^{p-2} .
\end{aligned}
$$

The $Q_{1-4}$ can be self-consistently set to zero, as they essentially are the correlation functions for the auxiliary MSR fields; besides this, it is immediate to see that the second term in the (A6) contains the sum of all the response functions for the system $Q_{9-12}$, multiplied for a sum of the same functions with inverted times $Q_{12-16}$; thus, since every response function $R\left(t, t^{\prime}\right)$ is zero for $t<t^{\prime}$ because of causality, the resulting product vanishes and the $\lambda_{5-9}$ are all equal to zero.

Using the saddle-point equations, we can eliminate the $\lambda_{\mathrm{s}}$ and replace the $Q \mathrm{~s}$ in the saddle point with the correlation functions,

$$
\begin{aligned}
& Q_{5}^{S P}\left(t, t^{\prime}\right) \equiv C_{\sigma \sigma}\left(t, t^{\prime}\right), \\
& Q_{6}^{S P}\left(t, t^{\prime}\right) \equiv C_{\sigma \tau}\left(t, t^{\prime}\right), \\
& Q_{7}^{S P}\left(t, t^{\prime}\right) \equiv C_{\tau \sigma}\left(t, t^{\prime}\right), \\
& Q_{8}^{S P}\left(t, t^{\prime}\right) \equiv C_{\tau \tau}\left(t, t^{\prime}\right),
\end{aligned}
$$

and the response functions of the system

$$
\begin{gathered}
Q_{13}^{S P}\left(t, t^{\prime}\right) \equiv-R_{\sigma \sigma}\left(t, t^{\prime}\right)=Q_{9}^{S P}\left(t^{\prime}, t\right) \\
Q_{14}^{S P}\left(t, t^{\prime}\right) \equiv-R_{\sigma \tau}\left(t, t^{\prime}\right)=Q_{10}^{S P}\left(t^{\prime}, t\right), \\
Q_{15}^{S P}\left(t, t^{\prime}\right) \equiv-R_{\tau \sigma}\left(t, t^{\prime}\right)=Q_{11}^{S P}\left(t^{\prime}, t\right) \\
Q_{16}^{S P}\left(t, t^{\prime}\right) \equiv-R_{\tau \tau}\left(t, t^{\prime}\right)=Q_{12}^{S P}\left(t^{\prime}, t\right) .
\end{gathered}
$$

If we now define

$$
K_{p} \equiv \frac{J^{2} p}{2^{2 p+1}}
$$

and

$$
\begin{aligned}
\mathcal{C} & =C_{\sigma \sigma}+C_{\sigma \tau}+C_{\tau \sigma}+C_{\tau \tau}, \\
\mathcal{R} & =R_{\sigma \sigma}+R_{\sigma \tau}+R_{\tau \sigma}+R_{\tau \tau},
\end{aligned}
$$

we can rewrite the averaged generating functional in the following way

$$
\begin{aligned}
\overline{Z[0]}= & \int \mathcal{D} \sigma \mathcal{D} \hat{\sigma} \mathcal{D} \tau \mathcal{D} \hat{\tau} \exp \left[-\frac{1}{2} \int d t d t^{\prime}\left(\hat{\boldsymbol{\sigma}} D_{0} \hat{\boldsymbol{\sigma}}+\hat{\boldsymbol{\tau}} D_{0} \hat{\boldsymbol{\tau}}\right)\right] \exp \left[-\frac{1}{2} \int d t d t^{\prime} K_{p}(\hat{\boldsymbol{\sigma}}+\hat{\boldsymbol{\tau}}) \mathcal{C}\left(t, t^{\prime}\right)^{p-1}(\hat{\boldsymbol{\sigma}}+\hat{\boldsymbol{\tau}})\right] \\
& \times \exp \int d t i \hat{\boldsymbol{\sigma}} \cdot\left[\dot{\boldsymbol{\sigma}}+\mu \boldsymbol{\sigma}+\frac{(D-T \log 2)}{2} \boldsymbol{\tau}-K_{p}(p-1) \int d t^{\prime} \mathcal{R}\left(t, t^{\prime}\right) \mathcal{C}\left(t, t^{\prime}\right)^{p-2}\left(\boldsymbol{\sigma}\left(t^{\prime}\right)+\boldsymbol{\tau}\left(t^{\prime}\right)\right)\right] \\
& \times \exp \int d t i \hat{\boldsymbol{\tau}} \cdot\left[\dot{\boldsymbol{\tau}}+\nu \boldsymbol{\tau}+\frac{(D-T \log 2)}{2} \boldsymbol{\sigma}-K_{p}(p-1) \int d t^{\prime} \mathcal{R}\left(t, t^{\prime}\right) \mathcal{C}\left(t, t^{\prime}\right)^{p-2}\left(\boldsymbol{\sigma}\left(t^{\prime}\right)+\boldsymbol{\tau}\left(t^{\prime}\right)\right)\right]
\end{aligned}
$$

as in [55], this functional is now local in space (i.e. refers to single sites only) but nonlocal in time; we can also see that this expression is quite similar to the one we had in equation (A1): we have the quadratic terms in $\hat{\boldsymbol{\sigma}}$ and $\hat{\boldsymbol{\tau}}$, containing the noise correlators, and the linear ones containing the equations of motion (16a), (16b) themselves.

\section{Equations for the correlation and the response}

Once the effective generating functional has been computed, the self-consistency equations for the correlation and response functions can be readily derived. For the $C_{\sigma \sigma}$ we just use the basic definition

$$
\frac{\partial C_{\sigma \sigma}\left(t_{1}, t_{2}\right)}{\partial t_{1}}=\left\langle\dot{\sigma}\left(t_{1}\right) \sigma\left(t_{2}\right)\right\rangle
$$

where $\langle\cdot\rangle$ denotes the average over the gaussian thermal noises $\xi(t)$ and $\zeta(t)[64]$; using the (16a), we get

$$
\begin{aligned}
\frac{\partial C_{\sigma \sigma}\left(t_{1}, t_{2}\right)}{\partial t_{1}}= & -\mu\left(t_{1}\right) C_{\sigma \sigma}\left(t_{1}, t_{2}\right)-\frac{(D-T \log 2)}{2} C_{\tau \sigma}\left(t_{1}, t_{2}\right) \\
& +K_{p}(p-1) \int d t^{\prime} \mathcal{R}\left(t, t^{\prime}\right) \mathcal{C}\left(t, t^{\prime}\right)^{p-2} \\
& \times\left(C_{\sigma \sigma}\left(t^{\prime}, t_{2}\right)+C_{\tau \sigma}\left(t^{\prime}, t_{2}\right)\right)+\left\langle\xi\left(t_{1}\right) \sigma\left(t_{2}\right)\right\rangle .
\end{aligned}
$$

The last term has to be computed using directly the general definition of an MSR generating functional (see [52] for details). First we write

$$
\bar{Z}=z^{N}
$$

where the $z$ is the reduced generating functional for a single couple of dynamic variables $(\sigma, \tau)$ only. With this definition we can write $\left\langle\xi\left(t_{1}\right) \sigma\left(t_{2}\right)\right\rangle$ as (to lighten the no- 
tation, we omit the time contractions).

$$
\begin{aligned}
\left\langle\xi\left(t_{1}\right) \sigma\left(t_{2}\right)\right\rangle & =\left.(-i) \frac{\delta}{\delta j\left(t_{1}\right)}\right|_{j=0} \int \delta \xi \delta \zeta \delta \sigma \delta \hat{\sigma} \delta \tau \delta \hat{\tau} \\
& \times \sigma\left(t_{2}\right) \exp \left[-\frac{1}{2}\left(\xi G_{\xi \xi} \xi+2 \xi G_{\xi \zeta} \zeta+\zeta G_{\zeta \zeta} \zeta\right)\right] \\
& \times \exp [i(-\xi \hat{\sigma}+\xi j-\zeta \hat{\tau})] \exp [\mathcal{L}]
\end{aligned}
$$

where the probability distribution of the noise has been made explicit by defining the inverse of the correlation matrix

$$
\begin{aligned}
G & \equiv D^{-1}, \\
D_{\xi \xi}\left(t, t^{\prime}\right) & \equiv\left\langle\xi(t) \xi\left(t^{\prime}\right)\right\rangle, \\
D_{\zeta \zeta}\left(t, t^{\prime}\right) & \equiv\left\langle\zeta(t) \zeta\left(t^{\prime}\right)\right\rangle, \\
D_{\xi \zeta}\left(t, t^{\prime}\right) & \equiv\left\langle\xi(t) \zeta\left(t^{\prime}\right)\right\rangle .
\end{aligned}
$$

and all the terms that don't contain the noise fields (the time-derivatives of the dynamic variables, the Lagrange multipliers, the convolutions with the response and correlation functions) have been cropped in $\mathcal{L}$.

The functional integral over the noise is a standard Gaussian integral with a linear term, so it can be readily performed, yielding

$$
\begin{aligned}
\int \delta \sigma \delta \hat{\sigma} \delta \tau \delta \hat{\tau} & \sigma\left(t_{2}\right) \exp \left\{-\frac{1}{2}\left[(-\hat{\sigma}+j) D_{\xi \xi}(-\hat{\sigma}+j)\right.\right. \\
& \left.\left.+2(-\hat{\sigma}+j) D_{\xi \zeta}(-\hat{\tau})+\hat{\tau} D_{\zeta \zeta} \hat{\tau}\right]\right\} \exp [\mathcal{L}]
\end{aligned}
$$

now, by taking the functional derivative with respect to $j\left(t_{i}\right)$ and multiplying by $-i$ we get

$$
\begin{aligned}
\left\langle\xi\left(t_{1}\right) \sigma\left(t_{2}\right)\right\rangle= & \int \delta \sigma \delta \hat{\sigma} \delta \tau \delta \hat{\tau} \int d t^{\prime}\left[D_{\xi \xi}\left(t_{1}, t^{\prime}\right)(-i) \sigma\left(t_{2}\right) \hat{\sigma}\left(t^{\prime}\right)\right. \\
& \left.+D_{\xi \zeta}\left(t_{1}, t^{\prime}\right)(-i) \sigma\left(t_{2}\right) \hat{\tau}\left(t^{\prime}\right)\right] e^{S(\sigma, \tau, \hat{\sigma}, \hat{\tau})} \\
= & \int d t^{\prime}\left[D_{\xi \xi}\left(t_{1}, t^{\prime}\right) R_{\sigma \sigma}\left(t_{2}, t^{\prime}\right)\right. \\
& \left.+D_{\xi \zeta}\left(t_{1}, t^{\prime}\right) R_{\sigma \tau}\left(t_{2}, t^{\prime}\right)\right]
\end{aligned}
$$

where $e^{S(\sigma, \tau, \hat{\sigma}, \hat{\tau})}$ is the probability distribution for the dynamic and auxiliary variables induced by the probability distribution for the noise[54].

By using this result, and recalling the definition (17a) for the correlation matrix, we can finally write the equation for the $C_{\sigma \sigma}$

$$
\begin{aligned}
\frac{\partial C_{\sigma \sigma}\left(t_{1}, t_{2}\right)}{\partial t_{1}}= & -\mu\left(t_{1}\right) C_{\sigma \sigma}\left(t_{1}, t_{2}\right) \\
& -\frac{(D-T \log 2)}{2} C_{\tau \sigma}\left(t_{1}, t_{2}\right) \\
& +K_{p}(p-1) \int_{-\infty}^{t_{1}} d t^{\prime} \mathcal{R}\left(t, t^{\prime}\right) \mathcal{C}\left(t, t^{\prime}\right)^{p-2} \\
& \times\left(C_{\sigma \sigma}\left(t^{\prime}, t_{2}\right)+C_{\tau \sigma}\left(t^{\prime}, t_{2}\right)\right) \\
& +2 T R_{\sigma \sigma}\left(t_{2}, t_{1}\right)+K_{p} \int_{-\infty}^{t_{2}} d t^{\prime} \mathcal{C}\left(t, t^{\prime}\right)^{p-1} \\
& \times\left(R_{\sigma \sigma}\left(t_{2}, t^{\prime}\right)+R_{\sigma \tau}\left(t_{2}, t^{\prime}\right)\right)
\end{aligned}
$$

the term proportional to the $R_{\sigma \sigma}$ is zero since we assume $t_{1}>t_{2}$. The same method can be applied to the remaining correlation functions.

For what concerns response functions, it can be easily proven that $[52,65]$

$$
\left\langle\frac{\delta \sigma\left(t_{1}\right)}{\delta \xi\left(t_{2}\right)}\right\rangle=\frac{\delta\left\langle\sigma\left(t_{1}\right)\right\rangle}{\delta h\left(t_{2}\right)}
$$

which in turn implies

$$
\frac{\partial R_{\sigma \sigma}\left(t_{1}, t_{2}\right)}{\partial t_{1}}=\frac{\partial}{\partial t_{1}}\left\langle\frac{\delta \sigma\left(t_{1}\right)}{\delta \xi\left(t_{2}\right)}\right\rangle=\left\langle\frac{\delta \dot{\sigma}\left(t_{1}\right)}{\delta \xi\left(t_{2}\right)}\right\rangle,
$$

and so

$$
\begin{aligned}
\frac{\partial R_{\sigma \sigma}\left(t_{1}, t_{2}\right)}{\partial t_{1}}= & -\mu\left(t_{1}\right)\left\langle\frac{\delta \sigma\left(t_{1}\right)}{\delta \xi\left(t_{2}\right)}\right\rangle \\
& -\frac{(D-T \log 2)}{2}\left\langle\frac{\delta \tau\left(t_{1}\right)}{\delta \xi\left(t_{2}\right)}\right\rangle \\
& +K_{p}(p-1) \int_{-\infty}^{t_{1}} d t^{\prime} \mathcal{R}\left(t, t^{\prime}\right) \mathcal{C}\left(t, t^{\prime}\right)^{p-2} \\
& \times\left[\left\langle\frac{\delta \sigma\left(t^{\prime}\right)}{\delta \xi\left(t_{2}\right)}\right\rangle+\left\langle\frac{\delta \tau\left(t^{\prime}\right)}{\delta \xi\left(t_{2}\right)}\right\rangle\right]+\left\langle\frac{\delta \xi\left(t_{1}\right)}{\delta \xi\left(t_{2}\right)}\right\rangle,
\end{aligned}
$$

The other equations can be obtained by trivially generalizing the (A14).

Let us make an important remark. The last term in the (A15) (given by the functional derivative of the noise with respect to itself) is a Dirac delta, which assures that

$$
\lim _{t_{1} \rightarrow t_{2}^{+}} R_{\sigma \sigma}\left(t_{1}, t_{2}\right)=1 \text {. }
$$

The same applies to $R_{\tau \tau}$. On the other hand, the equation for the $R_{\sigma \tau}$ is the following

$$
\begin{aligned}
\frac{\partial R_{\sigma \tau}\left(t_{1}, t_{2}\right)}{\partial t_{1}}= & -\mu\left(t_{1}\right) R_{\sigma \tau}\left(t_{1}, t_{2}\right)-\frac{(D-T \log 2)}{2} R_{\sigma \sigma}\left(t_{1}, t_{2}\right) \\
& +K_{p}(p-1) \int_{-\infty}^{t_{1}} d t^{\prime} \mathcal{R}\left(t, t^{\prime}\right) \mathcal{C}\left(t, t^{\prime}\right)^{p-2} \\
& \left(R_{\sigma \tau}\left(t^{\prime}, t_{2}\right)+R_{\sigma \sigma}\left(t^{\prime}, t_{2}\right)\right)
\end{aligned}
$$


which indeed lacks the Dirac delta, implying

$$
\lim _{t_{1} \rightarrow t_{2}^{+}} R_{\sigma \tau}\left(t_{1}, t_{2}\right)=0
$$

which applies to the $R_{\tau \sigma}$ too. This relation is fundamental to recover the static equation for the density (see appendix A 4).

The last thing left is computing the expression for the Lagrange multipliers $\mu(t)$ and $\nu(t)$; since we introduced those terms to enforce the spherical constraints, their expression must be determined self-consistently from them,

$$
\begin{aligned}
& C_{\sigma \sigma}(t, t)=N \Rightarrow \frac{d C_{\sigma \sigma}(t, t)}{d t}=0, \\
& C_{\tau \tau}(t, t)=N \Rightarrow \frac{d C_{\tau \tau}(t, t)}{d t}=0 .
\end{aligned}
$$

By expressing these total derivatives in terms of the dynamical equations for the correlation functions, we get, for the $\mu(t)$

$$
\begin{aligned}
\mu(t)= & -\frac{(D-T \log 2)}{2}(2 n(t)-1) \\
& +K_{p}(p-1) \int_{-\infty}^{t} d t^{\prime} \mathcal{R}\left(t, t^{\prime}\right) \mathcal{C}\left(t, t^{\prime}\right)^{p-2}\left(C_{\sigma \sigma}\left(t^{\prime}, t\right)+C_{\tau \sigma}\left(t^{\prime}, t\right)\right) \\
& +T R_{\tau \tau}(t, t)+K_{p} \int_{-\infty}^{t} d t^{\prime} \mathcal{C}\left(t, t^{\prime}\right)^{p-1}\left(R_{\sigma \sigma}\left(t, t^{\prime}\right)+R_{\sigma \tau}\left(t, t^{\prime}\right)\right)=0
\end{aligned}
$$

and for the $\nu(t)$,

$$
\begin{aligned}
\nu(t)= & -\frac{(D-T \log 2)}{2}(2 n(t)-1) \\
& +K_{p}(p-1) \int_{-\infty}^{t} d t^{\prime} \mathcal{R}\left(t, t^{\prime}\right) \mathcal{C}\left(t, t^{\prime}\right)^{p-2}\left(C_{\tau \tau}\left(t^{\prime}, t\right)+C_{\sigma \tau}\left(t^{\prime}, t\right)\right) \\
& +T R_{\sigma \sigma}(t, t)+K_{p} \int_{-\infty}^{t} d t^{\prime} \mathcal{C}\left(t, t^{\prime}\right)^{p-1}\left(R_{\tau \tau}\left(t, t^{\prime}\right)+R_{\tau \sigma}\left(t, t^{\prime}\right)\right)=0
\end{aligned}
$$

These are the expressions for $\mu(t)$ and $\nu(t)$ that are to be plugged in the equations for the correlation and response functions.

From this point, the derivation proceeds by imposing the FDT to couple the response and correlation functions, and then using the TTI to manipulate the resulting expressions. Those steps are only a matter of standard algebra, so we shall not report them.

\section{The $d \rightarrow 1$ limit}

The Blume Capel p-spin spherical model is supposed to be indistinguishable for a plain PSM in the $d \rightarrow 1$ limit, since in this limit the sites are all filled-in. So, our equations are supposed to give back the classic dynamical equation for the PSM when $d \rightarrow 1$.

From equation (28), we can see that $C_{\text {diff }}(t)$ is automatically zero for all times when $d=1$. So we only need to take care of equation (27), but the problem here is more tricky. In fact, it is immediate to verify that by just sending $d$ to one in the (27), we get a wrong result. The problem is that the value of $d$ depends strongly on the point $(\mathrm{D}, \mathrm{T})$ of the phase plane by means of equation (12); indeed, if we invert the picture and we treat $d$ as a free parameter, we see that when $d \rightarrow 1$, then the abso- lute value of the crystal field $D$ tends to infinity. This is perfectly reasonable; when $D$ becomes lower and lower, the configurations with a good number of empty sites are energetically disadvantaged, and the partition function is dominated mainly by configurations with many filled-in sites, which become the only ones accessible to the system when $D \rightarrow-\infty$. Since $|D| \rightarrow \infty$ at the same time as $d$ approaches one, the latter limit is nontrivial and has to be taken carefully.

Therefore, in order to estimate the order of magnitude of $D$ as $d$ tends to one, we solve the (12) for $D$, obtaining

$D=\frac{3 d^{p+1}-3 d^{p}+d^{2} T^{2} \log 16-4 T^{2} d(\log 2-1)-2 T^{2}}{4(d-1) T d}$.

By plugging this into equation (27), we have

$$
\begin{aligned}
\dot{C}(t)= & {\left[T-\frac{T}{2 d}-T\right] C(t) } \\
& -\frac{J^{2} p(d)^{p-1}}{4 T} \int_{0}^{t} d u C^{p-1}(t-u) \dot{C}(u) ;
\end{aligned}
$$

by imposing $d=1$, this becomes

$$
\dot{C}(t)=-\frac{T}{2} C(t)-\frac{J^{2} p}{4 T} \int_{0}^{t} d u C^{p-1}(t-u) \dot{C}(u),
$$


which is the dynamical equation for the PSM $[52,53,55]$, apart from a factor $1 / 2$ in front of the r.h.s.. Its presence is due to the fact that in the limit $d \rightarrow 1, \sigma=\tau$ and so our model contains $2 N \sigma$ spins, while the variance of the $J$ couplings has the usual scaling for a system of $N$ spins. By taking the long time limit, we get

$$
\frac{q}{1-q}=\frac{p \beta^{2}}{2} q^{p-1}
$$

which yields the well-established values for the dynamic transition temperature $T_{d}$ and the dynamical overlap $q_{d}$

$$
q_{d}=\frac{p-2}{p-1} \quad T_{d}=\sqrt{\frac{p(p-2)^{p-2}}{2(p-1)^{p-1}}} .
$$

This shows that, since in the long time limit $\dot{C}(t)$ is always zero, the $1 / 2$ factor is completely irrelevant for what concerns the properties of the system at the transition point.

\section{The equation for the density}

In this work, we have stressed multiple times the fact that the static equation (12) is needed to solve the dynamics of the system, since the equilibrium value of $d$ is not known a priori. This is fine for all practical purposes, but clearly unsatisfying from the theoretical point of view, as the (12) has been derived using replica theory in a static scenario. It is then necessary to show that equation (12) can be derived also in the MSR formalism in order to get a completely auto-consistent theoretical picture.

Our starting point is a trivial rephrasing of relation (3)

$$
n_{i}(t)=\frac{\sigma_{i}(t) \tau_{i}(t)+1}{2} .
$$

We sum this relation over lattice sites, divide by the system size $N$ and then take the thermal average on both sides. We get the following relation between the density and the $\sigma \tau$ correlation function

$$
\langle d(t)\rangle=\frac{C_{\sigma \tau}(t, t)+1}{2} .
$$

By taking the total derivative with respect to time we get

$$
\begin{aligned}
\frac{d\langle d(t)\rangle}{d t} & =\frac{1}{2} \frac{d C(t, t)}{d t} \\
& =\left.\frac{1}{2}\left(\frac{\partial C_{\sigma \tau}\left(t_{1}, t_{2}\right)}{\partial t_{1}}+\frac{\partial C_{\sigma \tau}\left(t_{1}, t_{2}\right)}{\partial t_{2}}\right)\right|_{t_{1}=t_{2}=t},
\end{aligned}
$$

which can be then rewritten in terms of the dynamical equations for the $C_{\sigma \tau}$

$$
\begin{aligned}
\frac{d\langle d(t)\rangle}{d t} & =-\mu(t)(2\langle d(t)\rangle-1)-\left(\frac{D-T \log 2}{2}\right) \\
& +\frac{J^{2} p(p-1)}{2^{2 p+1}} \int_{-\infty}^{t} d t^{\prime} \mathcal{R}\left(t, t^{\prime}\right) \mathcal{C}\left(t, t^{\prime}\right)^{p-2} \\
& \times\left(C_{\sigma \tau}\left(t^{\prime}, t\right)+C_{\tau \tau}\left(t^{\prime}, t\right)\right)+2 T R_{\tau \sigma}(t, t) \\
& +\frac{J^{2} p}{2^{2 p+1}} \int_{-\infty}^{t} d t \mathcal{C}^{p-1}\left(t, t^{\prime}\right)\left(R_{\tau \sigma}\left(t, t^{\prime}\right)+R_{\tau \tau}\left(t, t^{\prime}\right)\right) .
\end{aligned}
$$

Now, given that $\lim _{t_{1} \rightarrow t_{2}^{+}} R_{\sigma \tau}\left(t_{1}, t_{2}\right)=0$, and using the TTI and FDT, we get

$$
\begin{aligned}
\frac{d\langle d(t)\rangle}{d t}= & -\mu(t)(2 d(t)-1)-\left(\frac{D-T \log 2}{2}\right) \\
& -\frac{J^{2} p^{2}}{2^{2 p+2} T} \int_{0}^{\infty} \mathcal{C}(u)^{p-1} \dot{\mathcal{C}}(u) d u,
\end{aligned}
$$

where we have switched to the notation $\langle d(t)\rangle=d(t)$. The expression (A17) for the Lagrange multiplier can be manipulated in the same way, obtaining

$$
\begin{aligned}
\mu(t)= & -\frac{D-T \log 2}{2}(2 d(t)-1) \\
& -\frac{K_{p}}{2 T} \int_{0}^{\infty} d u \dot{\mathcal{C}}(u) \mathcal{C}(u)^{p-1}+T ;
\end{aligned}
$$

note that, since we are looking for a dynamical equation, we are now working with a time-dependent $d$.

We can now see that the integrals in both those expressions can be easily computed using the substitution formula. By doing so and than plugging the expression for $\mu$ in the equation we get

$$
\begin{aligned}
\dot{d}(t)= & (2 d(t)-1)^{2}\left(\frac{D-T \log 2}{2}\right)-\left(\frac{D-T \log 2}{2}\right) \\
& -\frac{J^{2} p}{2^{2 p+2} T}(4 d(t))^{p}(2 d(t)-1) \\
& +\frac{J^{2} p}{2^{2 p+2} T}(4 d(t))^{p}-T(2 d(t)-1),
\end{aligned}
$$

which, after some trivial algebra, leads to

$$
\dot{d}=2 d(d-1)(D-T \log 2)-\frac{p}{2 T} d^{p}(d-1)-T(2 d-1) .
$$

If we now assume the system to be at equilibrium at all times

$$
\dot{d}=0 \quad d(t)=d_{e q}
$$

the (A27) can be rewritten as

$$
\frac{p}{2} d^{p}(1-d)=T^{2}(2 d-1)+2\left(T D-T^{2} \log 2\right) d(1-d),
$$

which indeed is the static equation (12) for the density. This is the result we wanted. 
At this point, the reader has probably noticed the fact that, in this derivation, we have used the TTI and FDT, which are suited to an equilibrium situation, to derive a dynamical equation for the thermal average of a one-time observable, which is supposed to vary only in a nonequilibrium scenario.

This indeed is not a problem in itself. We could have embraced the equilibrium picture from the very beginning (i.e., setting $\frac{d C(t, t)}{d t}=0$ in the first place), and we would have anyway recovered the static equation for the density, which was the only result we needed for our dynamical study to be self-contained. However, the dynamic equation (A27) can be interesting to study in itself; by completing it with an initial value for the density $d(0)$, we obtain a Cauchy problem for which an unique solution is guaranteed to exist. We plot four such solutions in figures 7 and 8 for $(\mathrm{D}, \mathrm{T})=(2,0.2)$.
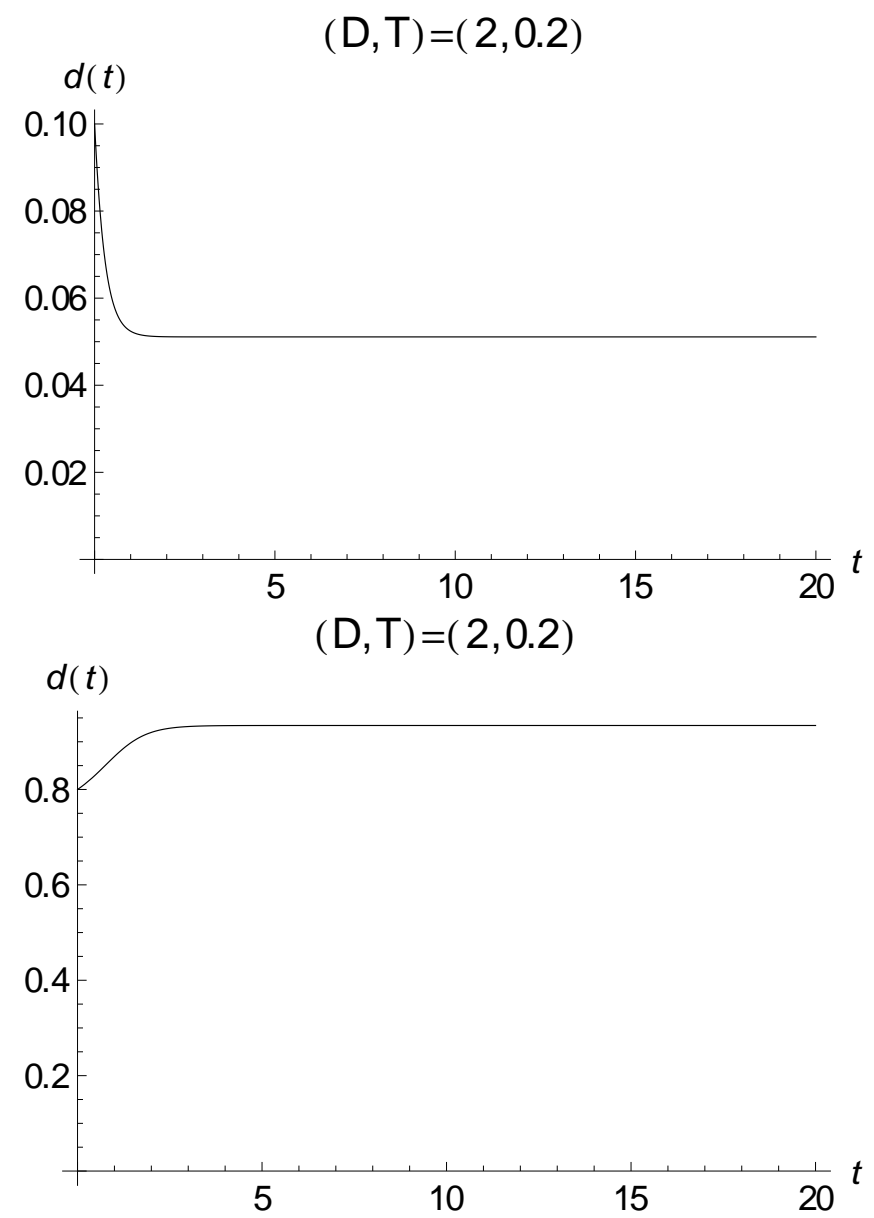

FIG. 7. Solutions of the $(\mathrm{A} 27)$ for $(D, T)=(0.2,2)$, with $d(0)=0.1$ (up), e $d(0)=0.8$ (down); both solutions converge rapidly to their respective equilibrium values, $d^{-}=0.051100$ and $d^{+}=0.934117$.

The plots show that, in the phase coexistence region, both equilibrium solutions correspond to an attractor for the dynamics, which is not surprising; where the solution actually ends up depends on the initial value $\mathrm{d}(0)$ that is chosen.

Besides this, it is interesting to notice that the unstable root $d_{\text {int }}$ of equation (12) (which, as we said in section II, is always to be discarded on grounds of replica stability arguments) is not an attractor for the dynamics, and actually is the critical value of $\mathrm{d}(0)$ that marks the separation point between the two attractors. Thus, the selection rule of section II for the value of the density is recovered from a dynamical point of view.
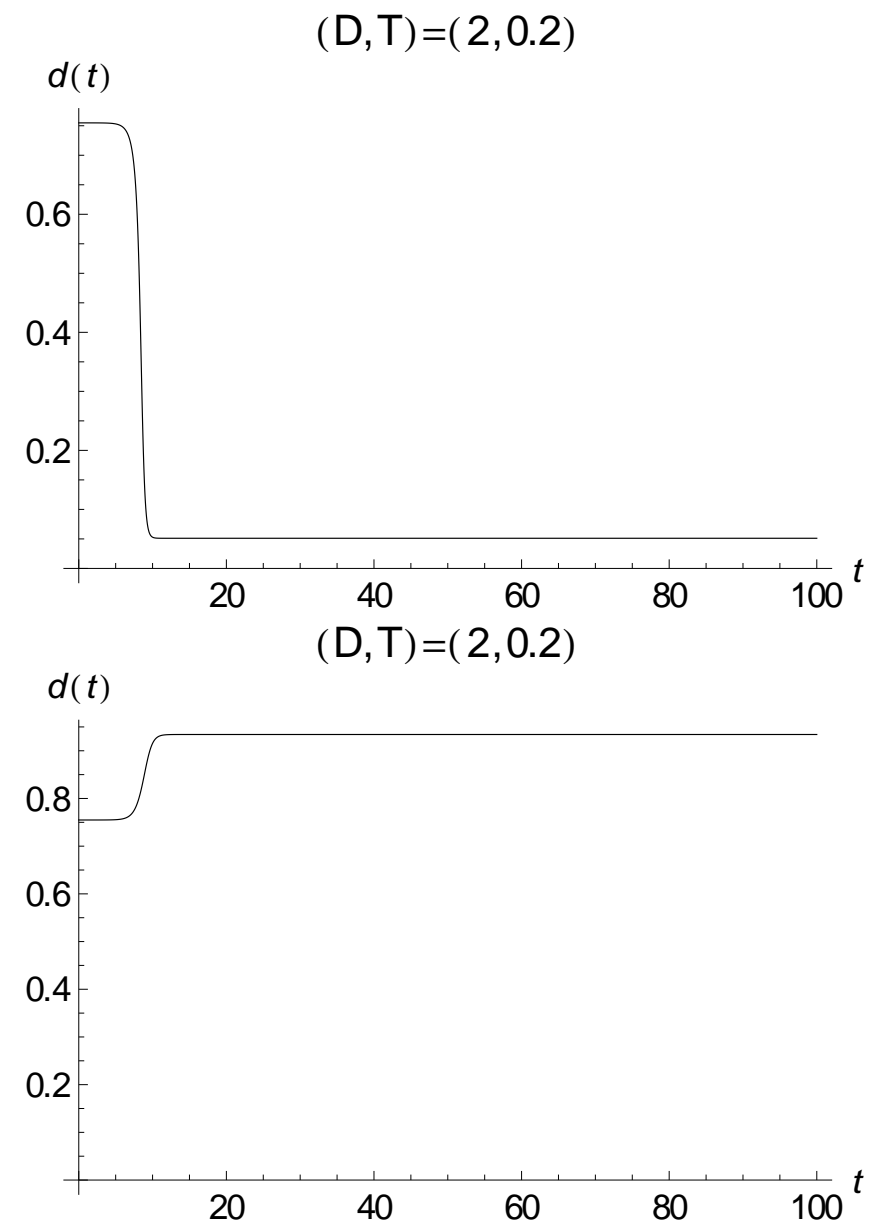

FIG. 8. Solutions of the (A27) for $(D, T)=(0.2,2)$, with $d(0)=0.754865$. slightly below $d_{\text {int }}$ (up), and $d(0)=$ 0.754867 slightly above $d_{\text {int }}($ down $) . d_{\text {int }}=0.754866$. The solution ends up in a different attractor depending on the choice of the initial value $d(0)$.

We conclude this section with a final remark. It may seem contradictory that the density $d(t)$ evolves in time, since it is a one-time quantity which is assumed to be constant in an equilibrium dynamics scenario. However, this is true only if the initial value $d(0)$ (which is completely arbitrary) is chosen different from both of the two equilibrium solutions of the static equation, which is exactly the contrary of what condition (26) requires.

Thus, the (26) can be seen both as a reasonable physical 
assumption, and as a self-consistency condition for the equilibrium dynamics.
[1] M. Blume, Physical Review 141, 517 (1966).

[2] H. Capel, Physica 32, 966 (1966).

[3] M. Blume, V. Emery, and R. Griffiths, Physical Review A 4, 1071 (1971).

[4] N. Schupper and N. M. Shnerb, Phys. Rev. Lett. 93, 037202 (2004).

[5] N. Schupper and N. M. Shnerb, Phys. Rev. B 72, 046107 (2005).

[6] S. K. Ghatak and D. Sherrington, J. Phys. C: Solid State Phys. 10, 3149 (1977).

[7] E. J. S. Lage and J. R. L. de Almeida, J. Phys. C: Solid State Phys. 15, L1187 (1982).

[8] P. J. Mottishaw and D. Sherrington, J. Phys. C: Solid State Phys. 18, 5201 (1985).

[9] M. Sellitto, M. Nicodemi, and J. J. Arenzon, J. Physique I 7, 945 (1997).

[10] G. R. Schreiber, Eur. Phys. J. B 9, 479 (1999).

[11] A. Crisanti and L. Leuzzi, Phys. Rev. Lett. 89, 237204 (2002).

[12] A. Crisanti and L. Leuzzi, Phys. Rev. B 70, 014409 (2004).

[13] U. Ferrari and L. Leuzzi, J. Stat. Mech. 2011, P12005 (2011).

[14] S. Rastogi, G. W. H. Höhne, and A. Keller, Macromolecules 32, 8897 (1999).

[15] A. L. Greer, Nature 404, 134 (2000).

[16] N. J. L. van Ruth and S. Rastogi, 37, 8191 (2004).

[17] M. Plazanet and et al., J. Chem. Phys. 121, 5031 (2004).

[18] E. Tombari and et al., J. Chem. Phys. 123, 051104 (2005).

[19] C. Ferrari and et al., J. Chem. Phys. 126, 124506 (2007).

[20] R. Angelini, G. Ruocco, and S. De Panfilis, Phys. Rev. E 79, 053502 (2009).

[21] A. Crisanti and L. Leuzzi, Phys. Rev. Lett. 95, 087201 (2005).

[22] M. Paoluzzi, L. Leuzzi, and C. A., Phys. Rev. Lett. 104, $120602(2010)$

[23] L. Leuzzi, M. Paoluzzi, and A. Crisanti, Phys. Rev. B 83, 014107 (2011).

[24] V. O. Ozcelik and A. N. Berker, Phys. Rev. E 78, 031104 (2008).

[25] F. Antenucci, A. Crisanti, and L. Leuzzi, Phys. Rev. E 90, 012112 (2014).

[26] F. Antenucci, A. Crisanti, and L. Leuzzi, J. Stat. Phys. 155, 909 (2014).

[27] E. Gardner, Nucl. Phys. B 257, 747 (1985).

[28] T. R. Kirkpatrick and D. Thirumalai, Phys. Rev. Lett. 58, 2091 (1987).

[29] T. R. Kirkpatrick and D. Thirumalai, Phys. Rev. B 36, 5388 (1987).

[30] A. Crisanti and H.-J. Sommers, Zeit. Phys. B 87, 341 (1992).

[31] A. Crisanti, L. Leuzzi, and T. Rizzo, Eur. Phys. J. B 36, 129 (2003).

[32] G. Parisi, Phys. Rev. Lett. 43, 1754 (1979).

[33] L. Leuzzi and T. A. Nieuwenhuizen, Thermodynamics of the glassy state (Taylor \& Francis, 2008).

[34] M. Müller, L. Leuzzi, and A. Crisanti, Phys. Rev. B 74,
134431 (2006).

[35] A. Crisanti, L. Leuzzi, G. Parisi, and T. Rizzo, Phys. Rev. B 70, 064423 (2004).

[36] A. Crisanti and H.-J. Sommers, J. Phys. I (France) 5, 805 (1995).

[37] F. Sciortino and P. Tartaglia, Advances in Physics 54, 471 (2005)

[38] K. Binder and K. W., Glassy Materials and Disordered Solids (World Scientific (Singapore), 2005).

[39] W. Götze, Complex dynamics of glass-forming liquids: a mode-coupling theory, Vol. 143 (Oxford University Press, 2009).

[40] J. H. Gibbs and E. A. Di Marzio, J. Chem. Phys. 28, 373 (1958).

[41] T. Hecksher, A. I. Nielsen, N. B. Olsen, and J. C. Dyre, Nature Phys. 4, 737741 (2008).

[42] J. P. Eckmann and I. Procaccia, Phys. Rev. E 78, 011503 (2008).

[43] H. Tanaka, Phys. Rev. Lett. 90, 05570 (2003).

[44] J. C. Martinez-Garcia, S. J. Rzoska, A. Drzozd-Rzoska, J. Martinez-Garcia, and J. C. Mauro, Sci. Rep. 4, 5160 (2014).

[45] M. Sellitto, Phys. Rev. B 73, 180202(R) (2006).

[46] T. H. Berlin and M. Kac, Phys. Rev. 86, 821 (1952).

[47] U. Ferrari, L. Leuzzi, G. Parisi, and T. Rizzo, Phys. Rev. B 86, 014204 (2012).

[48] R. Griffiths, Physica 33, 689 (1967).

[49] A. Caiazzo, A. Coniglio, and M. Nicodemi, Physical Review E 66, 046201 (2002).

[50] S. Franz and G. Parisi, J. Phys. I (France) 5, 1401 (1995).

[51] A. Crisanti, Nucl. Phys. B 796, 425 (2008).

[52] T. Castellani and A. Cavagna, J. Stat. Mech. P05012 (2005).

[53] A. Crisanti, H. Horner, and H.-J. Sommers, Zeitschr. Phys. B 92, 257 (1993).

[54] P. C. Martin, E. D. Siggia, and H. A. Rose, Phys. Rev. A 8, 423 (1973).

[55] T. R. Kirkpatrick and D. Thirumalai, Phys. Rev. B 36, 5388 (1987).

[56] In order to lighten the notation, in the following we shall use boldface to denote lattice site-dependent quantities. For example $\boldsymbol{\sigma}=\left(\sigma_{i}\right)_{i=1}^{N}$, and $\boldsymbol{\sigma} \cdot \boldsymbol{\sigma}=\sum_{i} \sigma_{i} \sigma_{i}$.

[57] Good introductions to the MSR formalism can be found in Refs. [52, 66].

[58] C. D. Dominicis, Phys. Rev. B 18, 4913 (1978).

[59] D. J. Gross and M. Mézard, Nucl. Phys. B 240, 431 (1984).

[60] M. Fuchs, W. Gotze, I. Hofacker, and A. Latz, Journal of Physics: Condensed Matter 3, 5047 (1991).

[61] L. Berthier, G. Biroli, J.-P. Bouchaud, W. Kob, K. Miyazaki, and D. R. Reichman, J. Chem. Phys. 126, 184504 (2007).

[62] A. Crisanti, L. Leuzzi, and M. Paoluzzi, Eur. Phys. J. E 34, 98 (2011).

[63] A. Crisanti and L. Leuzzi, J. Non-Cryst. Sol. 407, 110 (2015).

[64] The thermal noises $\xi$ and $\zeta$ have a non-diagonal correlation matrix, as it is immediate to see from the (17a). 
However, their probability distribution is still a Gaussian, albeit a non-factorized one. What is important is that the distribution can be factorized in terms where each one is relative to a single site.
[65] J.-P. Bouchaud, L. Cugliandolo, K. J., and M. Mézard, Physica A 226, 243 (1996).

[66] C. De Domicis and I. Giardina, Random fields and spin glasses (Cambridge University Press, 2006). 